\title{
A Brain without Brakes: Reduced Inhibition Is Associated with Enhanced but Dysregulated Plasticity in the Aged Rat Auditory Cortex
}

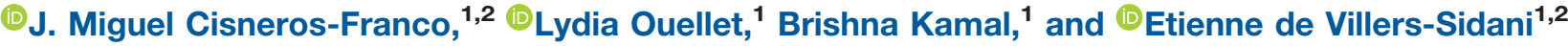

DOI:http://dx.doi.org/10.1523/ENEURO.0051-18.2018

${ }^{1}$ Department of Neurology and Neurosurgery, Montreal Neurological Institute, McGill University, Montreal QC H3A 2B4, Canada and ${ }^{2}$ Centre for Research on Brain, Language, and Music, Montreal QC H3G 2A8, Canada

\begin{abstract}
During early developmental windows known as critical periods (CPs) of plasticity, passive alterations in the quality and quantity of sensory inputs are sufficient to induce profound and long-lasting distortions in cortical sensory representations. With CP closure, those representations are stabilized, a process requiring the maturation of inhibitory networks and the maintenance of sufficient GABAergic tone in the cortex. In humans and rodents, however, cortical inhibition progressively decreases with advancing age, raising the possibility that the regulation of plasticity could be altered in older individuals. Here we tested the hypothesis that aging results in a destabilization of sensory representations and maladaptive dysregulated plasticity in the rat primary auditory cortex (A1). Consistent with this idea, we found that passive tone exposure is sufficient to distort frequency tuning in the $\mathrm{A} 1$ of older but not younger adult rats. However, we also found that these passive distortions decayed rapidly, indicating an ongoing instability of $A 1$ tuning in the aging cortex. These changes were associated with a decrease in GABA neurotransmitter concentration and a reduction in parvalbumin and perineuronal net expression in the cortex. Finally, we show that artificially increasing GABA tone in the aging A1 is sufficient to restore representational stability and improve the retention of learning.
\end{abstract}

Key words: Aging; auditory cortex; cortical plasticity; GABA; inhibition; training

\section{Significance Statement}

In this study, we examined brain plasticity in the auditory cortex of young adult and older adult rats in the context of different types of auditory stimulation and training. Surprisingly, older brains retained an equal or even higher potential for plasticity compared to young adults. In older brains, however, changes elicited by auditory stimulation and training were rapidly lost, suggesting that such increased plasticity might be detrimental, as the older brains were unable to consolidate these changes. This increased but poorly regulated plasticity was associated with a reduction in cortical inhibition, which normally maintains the stability of sensory representations in the young adult brain. Importantly, increasing inhibition artificially with clinically available drugs restored stability and improved the retention of learning.

\section{Introduction}

During early developmental epochs known as critical periods (CPs) of plasticity, passive exposure to environmental sounds profoundly shapes frequency tuning in the

Received February 1, 2018; accepted June 29, 2018; First published August 21, 2018.

The authors declare no competing financial interests. rat primary auditory cortex (A1; Hensch, 2005; de VillersSidani et al., 2007). On closure of the CP, these experience-dependent alterations are consolidated, and A1 tuning becomes relatively resistant to passive sound

Author Contributions: JMC and EdV designed research; JMC, LO, and BK performed research; JMC and EdV analyzed data; JMC and EdV wrote the paper. 
exposures. CP closure in sensory cortices is associated with the maturation of functional and structural inhibitory elements (Hensch, 2005; Fritschy and Panzanelli, 2014), including the maturation of parvalbumin positive $\left(\mathrm{PV}^{+}\right)$ interneurons (Kuhlman et al., 2013) and perineuronal nets (PNN; Wang and Fawcett, 2012). In the adult brain, plastic changes of the magnitude observed in the $\mathrm{CP}$ can be induced by down-regulating cortical inhibition (Fagiolini and Hensch, 2000) or disrupting elements involved in the stabilization of cortical representations such as PNNs (Pizzorusso et al., 2002; Carulli et al., 2010; Wang and Fawcett, 2012). Plastic changes of this magnitude otherwise still occur in the mature brain, but regulation mechanisms restrict them mainly to the context of behavior (Blake et al., 2006; Polley et al., 2006; Caroni et al., 2012). This tight gating of plasticity and resulting relative stability in the mature brain contribute to the consolidation and retention of new perceptuo-motor skills acquired through learning (Maffei and Turrigiano, 2008; Caroni et al., 2012).

Cortical inhibitory circuits are almost invariably affected by natural aging, as evidenced by a reduction of inhibitory tone and specific inhibitory interneurons such as $\mathrm{PV}^{+}$and somatostatin-positive $\left(\mathrm{SST}^{+}\right.$) cells in older brains (Caspary et al., 2008; Stanley et al., 2012; Ouellet and de Villers-Sidani, 2014). Given the importance of inhibitory processes in the regulation of plasticity and learning, it is reasonable to speculate that aging could have a significant impact on the mechanisms of learning in the brain (Caspary et al., 2008; Liguz-Lecznar et al., 2014). Loss of inhibition could lead to a state of cortical instability where sensory representations are easily distorted by nonspecific passive experiences, as is the case with the CP (Zhou et al., 2011). Such impairments could likely explain the noisy sensory processing and less effective learning and recovery observed in older rodents (Liguz-Lecznar et al., 2014) and humans (Boyke et al., 2008; Knoflach et al., 2012). Here, we tested these ideas using a combination of controlled passive pure tone exposure, pharmacological experiments, and behavioral training in young and old rats. We found that experience-dependent plasticity is paradoxically enhanced but unstable in old rats compared to young controls. Such instability was found even for relatively short 10 minute-long exposures and was paralleled by a reduction in the number of $\mathrm{PV}^{+}$cells and PNNs. Finally, we also demonstrated that this instability is associated with a more rapid decay of learning that can be reversed by artificially enhancing GABA tone in the brain.

Gouvernement du Canada I Canadian Institutes of Health Research (CIHR) grant MOP-133426 to EdV, and CIHR Vanier Canada Graduate Scholarship and CONACYT fellowship to JMC.

Acknowledgments: We are grateful to M. Thomas and P. Voss for useful comments on this manuscript.

Correspondence should be addressed to J. Miguel Cisneros-Franco, 3801 University Rm 753, Montreal, QC, H3A2B4, Canada. E-mail: mike.cisnerosfranco@mail.mcgill.ca.

DOI:http://dx.doi.org/10.1523/ENEURO.0051-18.2018

Copyright @ 2018 Cisneros-Franco et al.

This is an open-access article distributed under the terms of the Creative Commons Attribution 4.0 International license, which permits unrestricted use, distribution and reproduction in any medium provided that the original work is properly attributed.

\section{Materials and Methods}

All experimental procedures used in this study were approved by the Animal Care Committee and follow established guidelines. Twenty-three immature Long-Evans rats of either sex [age postnatal 10 days (P10) to P24], 24 Long-Evans young adult rats of either sex (age 6-8 mo), and 28 Long-Evans old adult rats of either sex (22-24 mo) were used for this study.

\section{Passive sound exposure}

The tone pip-exposed rats were housed for 1 or 2 consecutive weeks (24 h/d, $7 \mathrm{~d} / \mathrm{wk})$ in a sound attenuated chamber equipped with a speaker. The exposure sequences were generated using custom Matlab routines and contained repetitive trains of six 25-ms-long 5- or $10-\mathrm{kHz}$ pips with 5-ms cosine gates presented at a rate of 5 p.p.s. at an intensity of $70 \mathrm{~dB}$ SPL.

\section{Mapping the auditory cortex}

For A1 mapping, the rats were premedicated with dexamethasone $(0.2 \mathrm{mg} / \mathrm{kg})$ to minimize brain edema. They were anesthetized with ketamine/xylazine/acepromazine $(65 / 13 / 1.5 \mathrm{mg} / \mathrm{kg}$, i.p.) followed by a continuous delivery of isoflurane $1 \%$ in oxygen delivered via endotracheal intubation and mechanical ventilation. Vital signs were monitored using a MouseOx device (Starr Life Sciences). Body temperature was monitored with a rectal probe and maintained at $37^{\circ} \mathrm{C}$ with a homeothermic blanket system. The rats were held by the orbits in a custom-designed head holder, leaving the ears unobstructed. The cisterna magna was drained of cerebrospinal fluid to further minimize brain edema. The left temporalis muscle was reflected, auditory cortex (AC) was exposed, and the dura was resected. The cortex was maintained under a thin layer of silicone oil to prevent desiccation.

Cortical responses were recorded with 32-64-channel tungsten microelectrode arrays (Neuronexus). The microelectrode array was lowered orthogonally into the cortex to a depth of 470-600 \&micro;m (layers 4/5) where vigorous stimulus-driven responses were obtained. The extracellular neural action potentials were amplified, filtered $(0.3-5 \mathrm{kHz})$, sorted, and monitored on-line. Acoustic stimuli were generated using TDT System III (Tucker-Davis Technologies) and delivered in a free-field manner to the right ear through a calibrated speaker (Tucker-Davis Technologies). A software package (OpenEx; TuckerDavis Technologies) was used to generate acoustic stimuli, monitor cortical response properties on-line, and store data for off-line analysis. The evoked spikes of a single neuron or a small cluster of neurons were collected at each site.

Frequency-intensity receptive fields were reconstructed by presenting pure tones of 63 frequencies $(1-48 \mathrm{kHz}$; 0.1 -octave increments; 25 -ms duration; 5-ms ramps) at 8 sound intensities (0-70 dB SPL in 10-dB increments) to the contralateral ear at a rate of one stimulus per second. Ten-minute-long trains of 50 -ms tone pips were presented at 3 pulses per second at a sound intensity of 70 $\mathrm{dB}$ SPL. Each train had a commonly occurring frequency (standard) with a probability of occurrence of $80 \%$ and 5 
pseudo-randomly distributed oddball frequencies presented $20 \%$ of the time with no repetition. The oddball frequencies in the train had a constant separation of 1 octave.

\section{Electrophysiological data analysis}

The characteristic frequency (CF) of a cortical site was defined as the frequency at the tip of the $\mathrm{V}$-shaped tuning curve. For flat-peaked tuning curves, the CF was defined as the midpoint of the plateau at threshold. For tuning curves with multiple peaks, the CF was defined as the frequency at the most sensitive tip (i.e., with lowest threshold). The CF and threshold were determined using an automated routine developed in the Matlab environment (The MathWorks).

To generate A1 maps, Voronoi tessellation (a Matlab routine) was performed to create tessellated polygons with electrode penetration sites at their centers. Each polygon was assigned the characteristics (i.e., CF) of the corresponding penetration site. In this way, every point on the surface of the $A C$ was linked to the characteristics experimentally derived from its closest sampled cortical site. Primary AC (A1) was identified based on its rostralto-caudal tonotopy, reliable short-latency tone-evoked neuronal responses, and relatively sharp V-shaped RF. To examine A1 map plasticity, we compared the percentage of $A 1$ sites with CFs in 12 bins (width = octave) spanning the spectrum of presented tones.

Normalized responses to standard and oddball tones were obtained by dividing the average firing rate recorded in the $50 \mathrm{~ms}$ after the occurrence of each tone presentation by the average firing rate observed during the $50 \mathrm{~ms}$ after the first standard or oddball tone in the sequence. Asymptotes for standard and oddball responses were calculated by fitting exponential functions with a least squares method to the normalized response data from each recorded neuron. Simple linear regression of the normalized responses to the standard tone for the interval from event no. 150 to event no. 1200 was performed. The slope of the resulting best fit line was computed to determine the level of adaptation for each recorded site.

\section{Training}

Behavior was shaped in three phases. During the first phase, rats were trained to make a nose poke response to obtain a food reward. During the second phase, rats were trained to make a nose poke only after presentation of an auditory stimulus. During the third phase, the actual training program, rats were trained to make a nose poke only for the target stimulus (a 5-kHz pure tone) and not for a foil nontarget stimulus (10-kHz pure tone). The tones were presented at $60 \mathrm{~dB}$ SPL, stimulus presentation was randomized, and the probability of a target stimulus presentation was set at $20 \%$. Training was performed in an acoustically transparent operant training chamber $(60 \times 45 \times 35 \mathrm{~cm}$, length $\times$ width $\times$ height) contained within a sound-attenuated chamber. Sound presentation and response recording were performed using OpenEx software and RZ6 auditory processing hardware from Tucker-Davis Technology and delivered in a free field manner through a calibrated loudspeaker.

The intertrial interval was selected at random from a range of $4-6 \mathrm{~s}$. A rat's behavioral state at any point in time was classified as either "go" (producing a nose poke behavior) or "no-go." For a given trial, the rat could elicit one of four reinforcements produced by the combinations of responses (go or no-go) and stimulus properties (target or nontarget). Go responses within $5 \mathrm{~s}$ of a target were scored as a hit; a failure to respond within this time window was scored as a miss; a go response within $5 \mathrm{~s}$ of a nontarget stimulus was scored as a false positive; the absence of a response was scored as a withhold. A hit triggered the delivery of a food pellet. A miss or false positive initiated a 5-s time-out period during which time the house lights were turned off and no stimuli were presented. A withhold did not produce a reward or a time-out. Psychometric functions and stimulus target recognition indexes (D-prime) were calculated for each training session by plotting the percentage of go responses as a function of the total number of target stimuli (i.e., hit ratio) and the percentage of false positives as a function of the total number of foils (i.e., false-positive ratio). Learning curves were reconstructed by plotting the D-prime measure reached over successive days of training.

\section{GABA microdialysis}

Immediately after craniotomy (see Methods: Electrophysiology), a microdialysis probe (CMA 12 Microdialysis probe, Harvard Apparatus) was implanted in the AC using the stereotaxic coordinates (Paxinos and Watson, 2007): bregma AP, $-4.5 \mathrm{~mm}$; ML, $-7 \mathrm{~mm}$; DV, $4.5 \mathrm{~mm}$. The pump rate was set at $0.09 \mathrm{~mL} / \mathrm{h}$ (PHD ultra 4400 Syringe pump, Harvard Apparatus). Samples were manually collected and frozen at $-80^{\circ} \mathrm{C}$ until analysis with high performance liquid chromatography (Reinhoud et al., 2013).

\section{Immunohistochemistry}

Immediately following the end of recording sessions, rats received a high dose of pentobarbital $(85 \mathrm{mg} / \mathrm{kg}$ i.p.) and were perfused intracardially with $4 \%$ paraformaldehyde in $0.1 \mathrm{~m}$ PBS at $\mathrm{pH} 7.2$. Immediately after perfusion, rat brains were removed and placed in the same fixative overnight for further fixation and then transferred to a $30 \%$ sucrose solution, snap-frozen, and stored at $-80^{\circ} \mathrm{C}$ until sectioning. Fixed material was cut in the coronal plane along the tonotopic axis of $A 1$ on a freezing microtome at 40 \&micro; m. Tissue was incubated overnight at $4^{\circ} \mathrm{C}$ in either monoclonal or polyclonal antisera (for anti-PV: \#P3088 , dilution 1:10,000, Sigma-Aldrich; for PNN, fluorescein wisteria floribunda lectin \#FL-1351, dilution 1:200, Vector Laboratories). Tissue samples were always processed in pairs during immunostaining procedures to limit variables relative to antibody penetration, incubation time, and post-sectioning age/condition of tissue. A Zeiss LSM 510 Meta confocal microscope was used to assess fluorescence in the immunostained sections. Quantification of $\mathrm{PV}^{+}$cells and PNN optical density was performed in ImageJ (National Institutes of Health) and MetaMorph imaging software (Molecular Devices Systems), respectively. Digital images of $A 1$ cortical sections were taken with a $40 \times$ objective (Zeiss LSM 510). All quantification was assessed in 300-400-\&micro;m-wide A1 sectors (rostral, middle, caudal) extending from layer 1 to the underlying white matter by an experimenter blind to the age of 
Table 1. Statistical table.

\begin{tabular}{|c|c|c|c|}
\hline & Data structure & Type of test & Statistic and $p$ value \\
\hline a & Normal distribution & 2-way ANOVA; Tukey-Kramer test & $F(11,168)=14.84, p<0.001 ; p<0.001$ \\
\hline$b$ & Normal distribution & 2-way ANOVA; Tukey-Kramer test & $F(11,72)=4.02, p<0.001 ; p=0.87$ \\
\hline c & Normal distribution & 2-way ANOVA; Tukey-Kramer test & $F(11,72)=10.77, p<0.001 ; p<0.001$ \\
\hline d & Normal distribution & 2-way ANOVA; Tukey-Kramer test & $F(11,72)=13.13, p<0.001 ; p<0.001, p=0.35$ \\
\hline e & Normal distribution & 2-way ANOVA; Tukey-Kramer test & $F(11,72)=2.69, p=0.005 ; p=1, p=0.96$ \\
\hline$f$ & Normal distribution & 2-way ANOVA; Tukey-Kramer test & $F(11,72)=7.23, p<0.001 ; p=1, p<0.001$ \\
\hline$g$ & Normal distribution & 2-way ANOVA; Tukey-Kramer test & $F(11,132)=14.62, p=0 ; p<0.001, p=0.15$ \\
\hline $\mathrm{h}$ & Normal distribution & 2-way ANOVA; Tukey-Kramer test & $F(11,120)=12.58, p=0 ; p<0.001, p=0.1$ \\
\hline i & Nonnormal distribution & Wilcoxon rank-sum test & $z=-4.099, p=4.1 \times 10^{-5}$ \\
\hline j & Nonnormal distribution & Wilcoxon rank-sum test & $z=-3.187, p=0.0014$ \\
\hline $\mathrm{K}$ & Normal distribution & $t$ test & $t(579)=5.64, p<0.001$ \\
\hline I & Normal distribution & $t$ test & $t(395)=3.35, p=9 \times 10^{-4}$ \\
\hline $\mathrm{m}$ & Normal distribution & $t$ test & $t(750)=0.75, p=0.45$ \\
\hline$n$ & Normal distribution & $t$ test & $t(408)=0.64, p=0.52$ \\
\hline 0 & Normal distribution & $t$ test & $t(383)=2.55, p=0.011$ \\
\hline$p$ & Nonnormal distribution & Wilcoxon rank-sum test & $z=-4.4, p=1.1 \times 10^{-5}$ \\
\hline$q$ & Nonnormal distribution & Wilcoxon rank-sum test & $z=\times 2.46, p=0.013$ \\
\hline r & Normal distribution & $t$ test & $t(720)=5.29, p<0.001$ \\
\hline s & Normal distribution & $t$ test & $t(345)=2.1, p=0.03$ \\
\hline$t$ & Normal distribution & $t$ test & $t(690)=0.86, p=0.39$ \\
\hline $\mathrm{u}$ & Normal distribution & $t$ test & $t(308)=0.08, p=0.94$ \\
\hline$v$ & Normal distribution & $t$ test & $t(18)=2.32, p=0.032$ \\
\hline w & Normal distribution & 2-way ANOVA; Tukey-Kramer test & $F(11,72)=13.42, p<0.001 ; p=0.018$ \\
\hline $\mathrm{x}$ & Normal distribution & 2-way ANOVA; Tukey-Kramer test & $F(11,72)=6.57, p<0.001 ; p=0.004, p=0.41$ \\
\hline y & Normal distribution & $t$ test & $t(6)=5.02, p=0.002$ \\
\hline$z$ & Normal distribution & 2-way ANOVA; Tukey-Kramer test & $F(11,72)=6.68, p<0.001 ; p=0.01$ \\
\hline$a b$ & Normal distribution & 2-way ANOVA; Tukey-Kramer test & $F(11,72)=1.41, p=0.18$ \\
\hline ac & Normal distribution & 2-way ANOVA; Tukey-Kramer test & $F(11,72)=5.42, p<0.001 ; p=0.022$ \\
\hline ad & Normal distribution & $t$ test & $t(6)=2.53, p=0.04$ \\
\hline ae & Normal distribution & $t$ test & $t(6)=3.66, p=0.01$ \\
\hline af & Nonnormal distribution & Kruskal-Wallis test; Tukey-Kramer post hoc test & $H(4)=14.52, p=0.0058 ; p=0.52, p=0.011, p=0.96, p=0.97$ \\
\hline ag & Nonnormal distribution & Kruskal-Wallis test; Tukey-Kramer post hoc test & $H(4)=83.97, p<0.0001 ; p<0.0001, p<0.001, p=0.96, p=0.003$ \\
\hline ah & Nonnormal distribution & Kruskal-Wallis test; Tukey-Kramer post hoc test & $H(4)=13, p=0.011 ; p=0.82, p=0.005, p=0.99, p=0.99$ \\
\hline ai & Nonnormal distribution & Kruskal-Wallis test; Tukey-Kramer post hoc test & $H(4)=17.24, p=0.0017 ; p=0.48, p=0.04, p=0.004, p=0.8, p=0.99$ \\
\hline aj & Nonnormal distribution & Kruskal-Wallis test; Tukey-Kramer post hoc test & $H(4)=22.06, p<0.001 ; p=0.004, p=0.039, p=0.99, p=0.85$ \\
\hline
\end{tabular}

the animals. $\mathrm{PV}^{+}$cells were classified into four subclasses as follows: low-PV, $0-0.8 \times 10^{5}$; intermediate low-PV, 8-1.6 $\times 10^{5}$; intermediate high-PV, 1.6-2.4 $\times 10^{5}$; high-PV, $>2.4$ $\times 10^{5}$. PNNs were classified into four subclasses as follows: low PV, $0-1 \times 10^{4}$, intermediate low-PV, $1-2 \times 10^{5}$; intermediate high-PV, $2-3 \times 10^{5}$; high-PV, $>3 \times 10^{5}$.

\section{Statistical analysis}

For normally distributed data, statistical significance was assessed using unpaired two-tailed $t$ tests or two-way analysis of variance with Tukey post hoc correction for multiple comparisons. Wilcoxon rank-sum test or Kruskal-Wallis test with Tukey post hoc correction for multiple comparisons were used for nonparametric data analysis. Data are presented as mean \pm SEM or median \pm median absolute deviation (MAD). Superscript letters listed with $p$-values correspond to the statistical tests shown in Table 1.

\section{Results}

\section{Passive tone exposure induces significant shifts in A1 tuning}

Aging is characterized by a progressive reduction in cortical inhibition to levels akin to those observed during developmental critical periods (Caspary et al., 2008; Stan- ley et al., 2012; Ouellet and de Villers-Sidani, 2014; Stebbings et al., 2016). This raises the possibility that the old brain has in fact a higher plastic potential than its young adult counterpart. To test this hypothesis, we examined the effect of pure-tone exposure on spectral tuning in the aged $\mathrm{A} 1$. We exposed old adult rats (OA, 22-23 months old, $n=4$ ) to $5 \mathrm{kHz}$ tone pips for 1 week. For comparison, the same exposure was used in young adult (YA, 6-8 months old, $n=4$ ) and immature rats in their $\mathrm{CP}$ window (I, P10-P17, $n=8$; Fig. 1A). We then compared the proportion of $A 1$ neurons whose characteristic frequency was close to the exposure frequency. As expected, there was a clear effect of $5-\mathrm{kHz}$ tone exposure on the $\mathrm{CF}$ of immature rats (two-way ANOVA, exposure group $\times$ frequency bin, $\left.F(11,168)=14.84, p<0.001^{\mathrm{a}}\right)$. Such an exposure resulted in a significant overrepresentation of the exposure tone in A1 of immature rats (average \% difference in the proportion of recording sites tuned within octave of exposure tone, relative to control: $9.77 \pm$ $1.54 \%$ increase, $p<0.001^{\mathrm{a}}$, with Tukey-Kramer correction) but not in the young adult group $(F(11,72)=4.02$, $p<0.001 ; 3.84 \pm 1.3 \%$ increase, $p=0.87^{b}$, with TukeyKramer correction; Fig. 1B). Passive tone exposure, however, resulted in a significant overrepresentation of the 
A
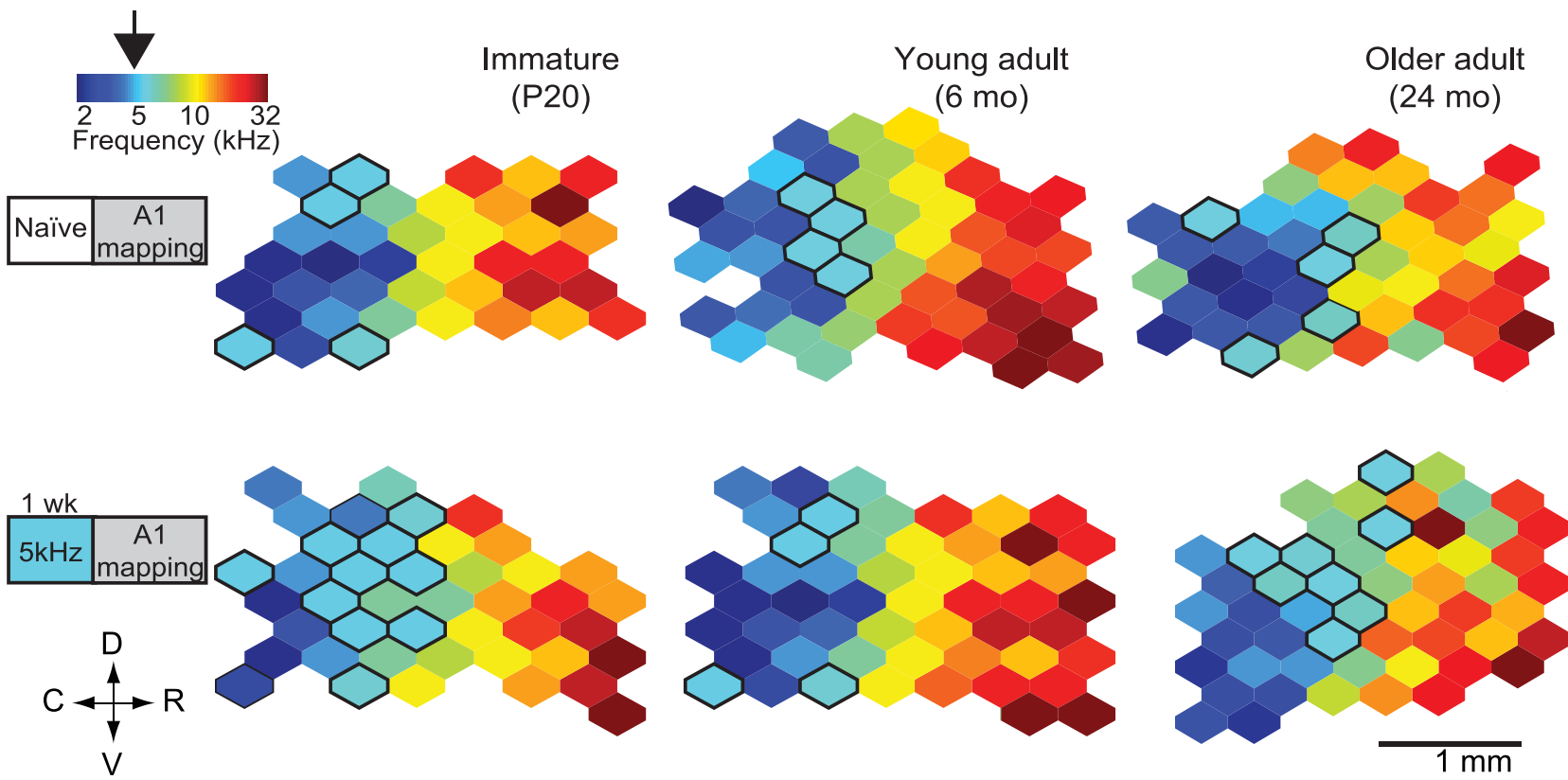

B

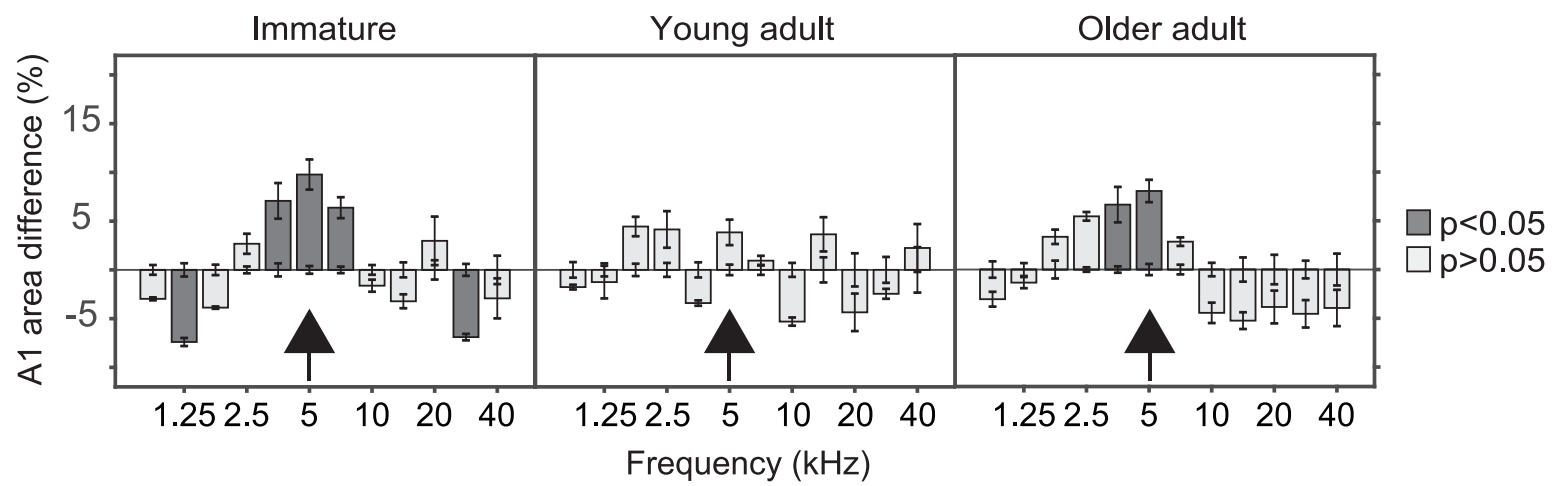

Figure 1. Passive sound exposure alters frequency tuning in the aged $A 1$. $\boldsymbol{A}$, Representative $A 1 \mathrm{CF}$ maps from naïve rats (top) and from rats exposed to $5-\mathrm{kHz}$ pure tones during 1 week (bottom). D, dorsal; $\mathrm{C}$, caudal; $\mathrm{R}$, rostral; $\mathrm{V}$, ventral. $\boldsymbol{B}$, Difference in frequency tuning between naïve and exposed rats expressed as A1 percentage area and separated by CF for immature, young adult, and old adult groups. Immature group: $n=8$, recorded sites $=389$; YA: $n=8$, recorded sites $=403$; OA: $n=8$, recorded sites $=382$; immature-exposed: $n=8$, recorded sites $=362$; YA-exposed: $n=4$, recorded sites $=177$; OA-exposed: $n=4$, recorded sites $=$ 168. Values shown are mean, two-way ANOVA with Tukey-Kramer correction.

exposure tone in the aged $\mathrm{A} 1$ group $(F(11,72)=10.77$, $p<0.001$, two-way ANOVA; $8.05 \pm 1.14 \%$ increase, $p<$ $0.001^{\mathrm{c}}$, with Tukey-Kramer correction).

To further document the extent of this tuning instability in older rats, we examined the effect of two consecutive pure-tone exposures over a 2-week period. Young $(n=4)$ and old $(n=4)$ adult rats were exposed to $10-\mathrm{kHz}$ tone pips during the first week and to $5-\mathrm{kHz}$ tone pips during the second week (Fig. 2A). This passive exposure protocol resulted in an overrepresentation of the second (5$\mathrm{kHz})$ exposure tone in $\mathrm{A} 1$ of aged rats $(F(11,72)=13.13$, $p<0.001$, two-way ANOVA; $5 \mathrm{kHz}: 8.81 \pm 1.7 \%$ increase, $p<0.001 ; 10 \mathrm{kHz}: 5.18 \pm 1.3 \%$ decrease, $p=$ $0.35^{\mathrm{d}}$, with Tukey-Kramer correction) but did not alter the frequency tuning map of young adults $(F(11,72)=2.69$, $p=0.005$, two-way ANOVA; $5 \mathrm{kHz}: 2.1 \pm 0.56 \%$ decrease, $p=1 ; 10 \mathrm{kHz}$ : $3.4 \pm 0.57 \%$ decrease, $p=0.96^{\mathrm{e}}$, with Tukey-Kramer correction; Fig. 2B).

\section{Enhancing cortical inhibition stabilizes frequency representation in the aged $\mathrm{A} 1$}

Having documented the existence of age-related tuning instability in $\mathrm{A} 1$, and considering the reduction of intracortical inhibition in sensory cortices associated with advanced age (Lehmann et al., 2012; Wang and Fawcett, 2012), we hypothesized that increasing GABAergic tone would restore the excitatory/inhibitory (E/I) balance and prevent further plastic changes elicited by passive tone exposure. To determine the effect of enhancing inhibition on frequency tuning stability in aged rats, we systemically administered the $\mathrm{GABA}_{\mathrm{A}}$ agonist diazepam (1 mg/kg i.p., twice a day; $n=4$ ) during two consecutive pure-tone exposures as described above $(10-\mathrm{kHz}$ pure tones for 1 week followed by $5-\mathrm{kHz}$ pure tones for 1 week; Fig. 2C). We found that diazepam administration resulted in an overrepresentation of the first $(10-\mathrm{kHz})$ rather than the second $(5-\mathrm{kHz})$ exposure tone $(F(11,72)=7.23, p<$ 

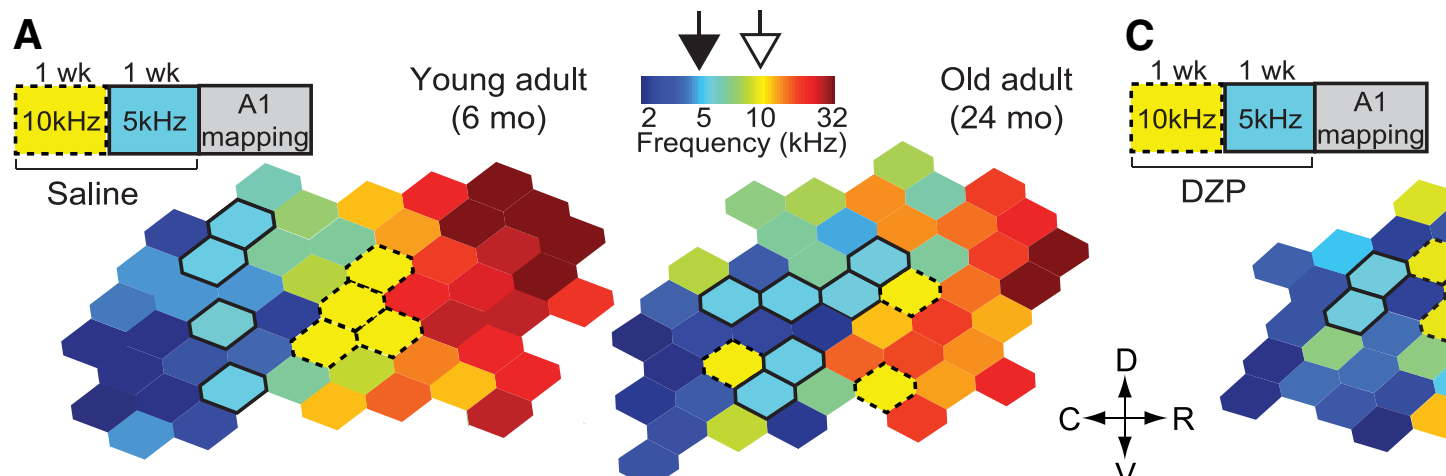

B

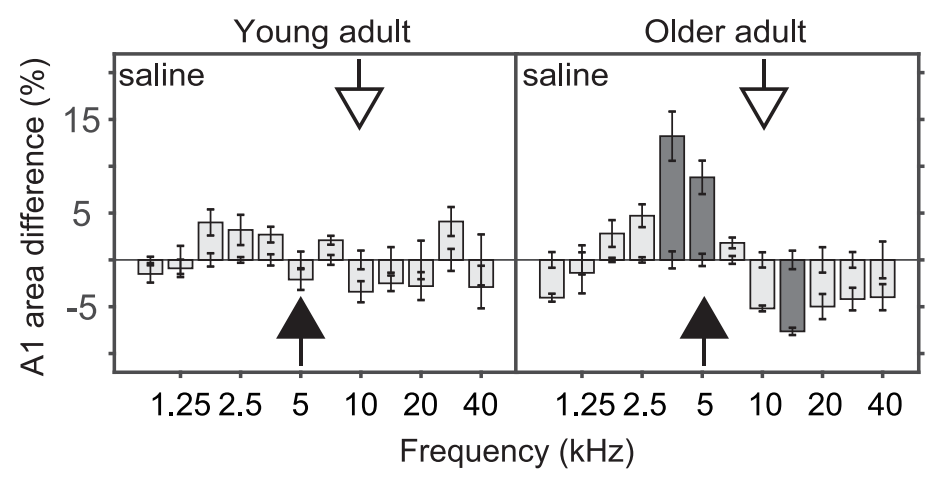

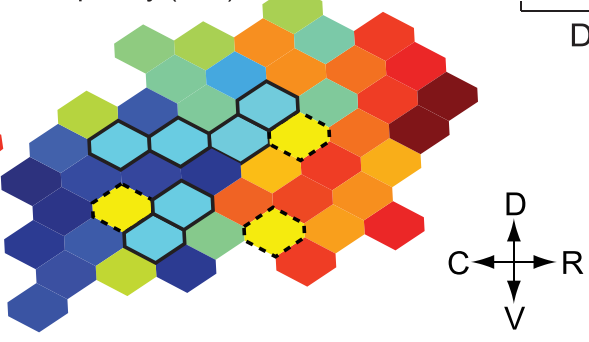

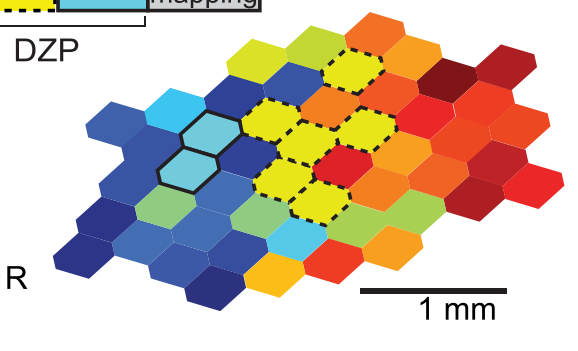

D

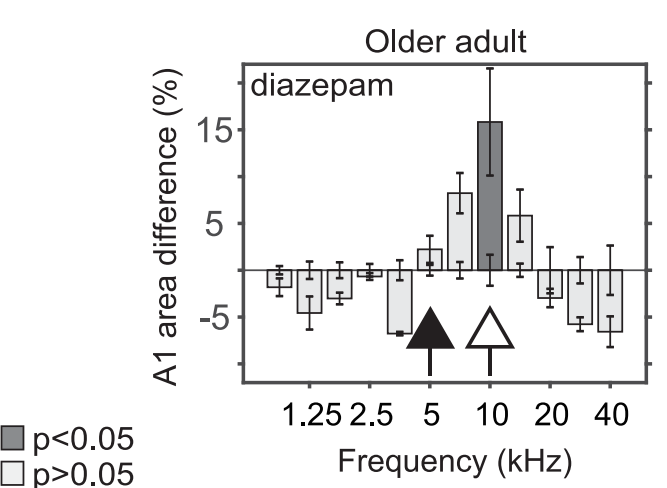

Figure 2. Restoration of inhibition stabilizes frequency representation in the aged $A 1$. Young and old adult rats were exposed to $10-\mathrm{kHz}$ pure tones for 1 week, followed immediately by exposure to $5-\mathrm{kHz}$ pure tones for 1 week. $\boldsymbol{A}$, Representative A1 CF maps from young (left) and old (right) adult rats that received sham (saline) intraperitoneal injections during the 2-week passive exposure period. $\boldsymbol{B}$, Difference in frequency tuning between naïve and saline-treated rats expressed as A1 percentage area and separated by CF. $\boldsymbol{C}$, Representative A1 CF map from an old adult rat that received diazepam (DZP) intraperitoneal injections during the 2-week passive exposure period. $\boldsymbol{D}$, Difference in frequency tuning between naïve and DZP-treated rats. To investigate whether sequential exposure to pure tones would have a similar effect in immature rats, 2-week exposures were conducted starting on P10 as described in Fig. 2-1. YA-saline group: $n=4$, recorded sites $=230$; OA-saline: $n=4$, recorded sites $=203$; OA-diazepam: $n=4$; recorded sites $=$ 218. Values shown are mean, two-way ANOVA with Tukey-Kramer correction. Conventions as in Fig. 1.

0.001 , two-way ANOVA; $5 \mathrm{kHz}: 2.23 \pm 1.45 \%$ increase, $p=1 ; 10 \mathrm{kHz}: 13.8 \pm 5.7 \%$ increase, $p<0.001^{\dagger}$, corrected with Tukey-Kramer test; Fig. 2D).

To investigate whether sequential exposure to pure tones would have a similar effect in immature animals as in old adult animals, we used a sequential 2-week exposure paradigm starting at $\mathrm{P} 10(5 \mathrm{kHz}$ pure tones for 1 week, followed by $10 \mathrm{kHz}$ pure tones for 1 week, $n=5$ ). Previous experiments have shown that passive tone exposure outside the CP for frequency tuning ( P10-P14) does not alter the A1 tonotopic map (de Villers-Sidani et al., 2007). For this reason, we predicted that such an exposure would result in an overrepresentation of the tone presented during the $\mathrm{CP}$-the first exposure toneregardless of any subsequent tone presentation. As expected, and in contrast to the results observed in the OA group, we observed plasticity in response to the first exposure tone $(F(11,132)=14.62, p=0$, two-way ANOVA; $5 \mathrm{kHz}: 13.27$ $\pm 3.4 \%$ increase, $p<0.001 ; 10 \mathrm{kHz}: 5.72 \pm 1.6 \%$ decrease, $p$ $=0.15^{\mathrm{g}}$, corrected with Tukey-Kramer test; Fig. 2-1A, left) in the vehicle (saline) condition.

Although treatment with diazepam accelerates the closing of the CP (Iwai et al., 2003), it does not prevent experience-dependent plasticity from taking place
(Hensch et al., 1998; Fagiolini and Hensch, 2000). In line with these observations, sequential tone exposure in immature rats treated with diazepam $<(n=4)$ resulted in a significant expansion of the tone presented during the span of the CP; i.e., the first exposure tone $(F(11,120)=$ $12.58, p<0.001$, two-way ANOVA; $5 \mathrm{kHz}: 11.93 \pm 3.6 \%$ increase, $p=0.018 ; 10 \mathrm{kHz}: 6.11 \pm 1.1 \%$ decrease, $p=$ $0.1^{\mathrm{h}}$, corrected with Tukey-Kramer test; Fig. 2-1A, $B$, right).

\section{Reversal of adaptation in the immature and aged A1}

Auditory neurons continuously monitor the environment, suppressing their response to repetitive sounds and making novel stimuli more salient (Ulanovsky et al., 2003; Malmierca et al., 2014). In the adult A1, such stimulus-specific adaptation prevents the overrepresentation of repetitive stimuli that drive plasticity during early development (Norena et al., 2006) and is also involved in the selection of A1 representations that should be selectively suppressed in the context of training (Froemke et al., 2013). With aging, however, receptive fields become less reliable across successive repetitions of the same set of stimuli (Turner et al., 2005). To examine the 
A

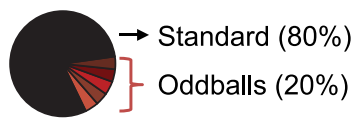

B

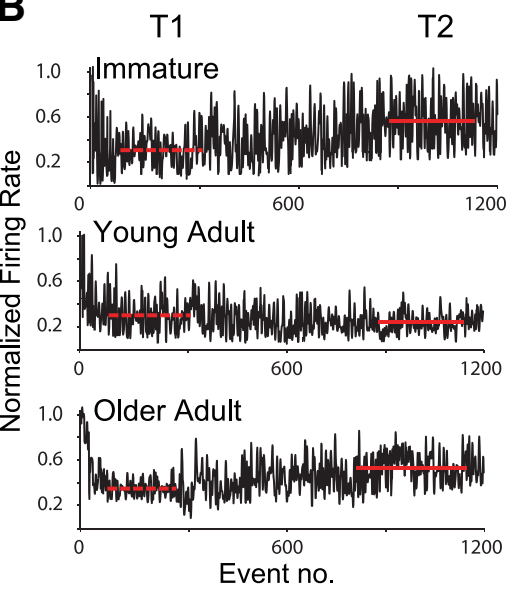

F

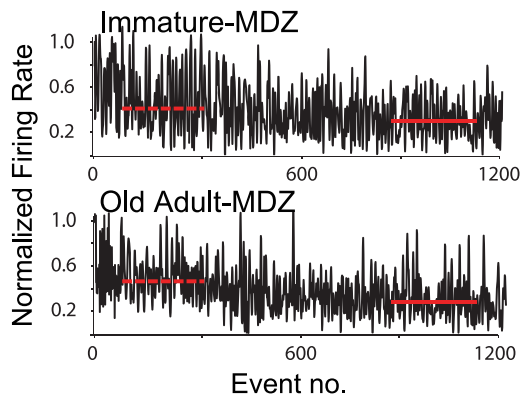

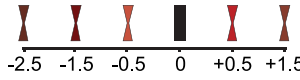

$\Delta$ Frequency (octaves)

C

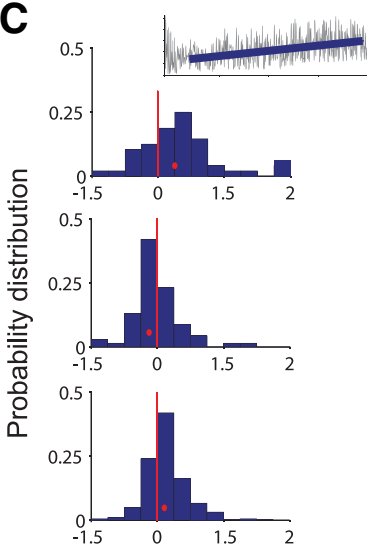

Slope of firing rate trace ( $\mathrm{n} \times 10-4)$

G

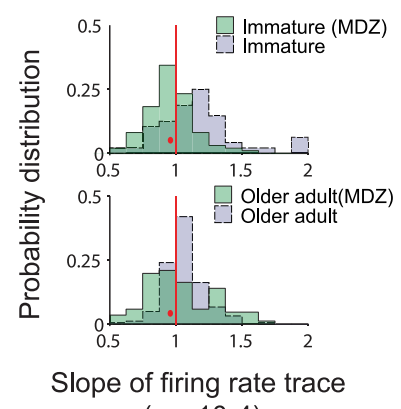

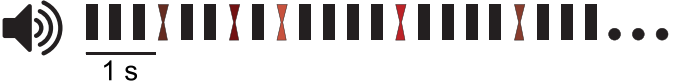
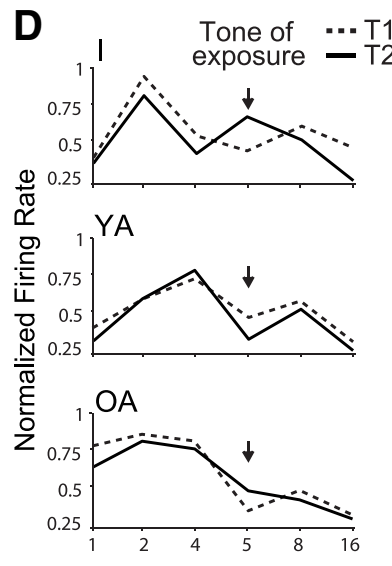

$\Delta$ Frequency $(\mathrm{KHz})$
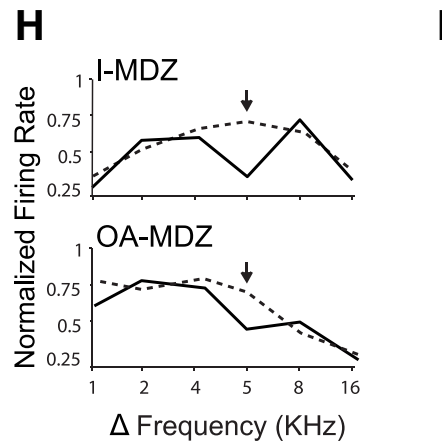

E

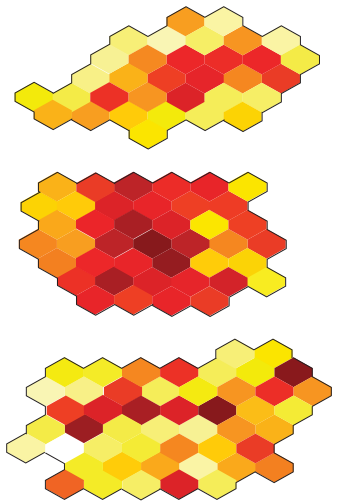

0.511 .5

$\mathrm{T} 2 / \mathrm{T} 1$ ratio

I
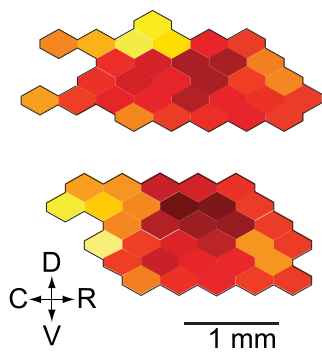

Figure 3. Improved adaptation in the immature and aged A1 following administration of the GABAA agonist midazolam. $\boldsymbol{A}$, Stimulation paradigm. Left, a standard (high-probability) tone was presented $80 \%$ of times. Five oddball (low-probability) tones distributed around the standard frequency (middle) were interspersed in the repetitive tone presentation (right). $\boldsymbol{B}$, Representative normalized responses of individual A1 neurons to a standard tone $(5$ or $12 \mathrm{kHz}$ at a repetition rate of $3 \mathrm{~Hz}$ ) as function of tone position in the stimulus sequence. Red horizontal lines represent the average normalized firing rate in response to the standard tone during two different intervals in the stimulus sequence: early (T1, event 100-300; dashed line), and late (T2, event 900-1100; solid line). Note that adaptation is reduced in both immature (I) and old adult rats. $\boldsymbol{C}$, Probability distribution plot of the slope of firing rate trace in response to the standard tone (interval from event no. 150-1200). Red dots denote the location of the median value for each group. Fig. 3-1 provides a summary of data related to adaptation in response to repetitive tones for all five groups. $\boldsymbol{D}$, Frequency tuning of representative A1 neurons during T1 (dashed line) and T2 (solid line). The normalized spike rate is plotted for the standard tone (arrow) and each of the five deviant tones. Note the acute change in tuning after standard-oddball presentation in I and OA rats. $\boldsymbol{E}$, Representative A1 activity maps depicting the change in firing rate at T2 relative to T1 (T2/T1 ratio of normalized firing rate). Warmer colors (white, yellow) denote neurons with reduced adaptation, notably in the I and OA groups. Same conventions apply for panels $\boldsymbol{F}-\boldsymbol{I}$, which show that midazolam improved adaptation and prevented changes in tuning in the immature and aged $A 1$. Immature group: $n=8$, recorded sites $=376$; YA: $n=4$, recorded sites $=205$; OA: $n=4$, recorded sites $=192$; I-MDZ: $n=8$, recorded sites $=346$; OA-MDZ: $n=4$, recorded sites $=155$.

extent to which aging $A 1$ neurons exhibit SSA, we used 10-min-long trains of pure tones (Fig. $3 A$ ).

As expected, we found a progressive decrease in A1 neuron responses to repetitive tones in younger adults (median slope of normalized response rate: YA, $-0.14 \pm$ $0.03 \times 10^{-4}$, number of recorded cortical sites $=205$ ) but an increase in responses to repetitive tones in the immature and old adult groups relative to YA (median slope of normalized response rate: I, $0.09 \pm 0.03 \times 10^{-4}, p=4.1$ $\times 10^{-5}, z=-4.099^{i}$, number of recorded cortical sites $=$
376; OA, $0.11 \pm 0.02 \times 10^{-4}, p=0.0014, z=-3.187^{\mathrm{j}}$, number of recorded cortical sites $=192$; Wilcoxon ranksum test; Fig. 3B, C).

Tuning stability in the same A1 neurons was examined by interspersing five oddball (low-probability) tones covering the hearing range during the repetitive (highprobability) tone presentation (see Methods). Using this method, coarse tuning curves could be constructed over two time intervals during the tone train exposure (T1, from 60 to $120 \mathrm{~s}$; T2, from 400 to $460 \mathrm{~s}$ ). On average, A1 
neurons in the immature and old adult groups exhibited a significant increase in response to the high-probability tone from T1 to T2, while the opposite was seen in the young adult group (change in normalized firing rate, T2 minus T1: YA, $-0.19 \pm 0.05 ; \mathrm{I}, 0.18 \pm 0.04, p<0.001$, $t(579)=5.64^{\mathrm{k}}$, relative to YA; OA, $0.15 \pm 0.09 p=9 \times$ $10^{-4}, t(395)=3.35^{1}$, relative to YA; $t$ test). Interestingly, the sum of responses to high and low probability tones remained constant in the immature and young adult groups, while it increased for the old adult group (difference in mean area under the curve between T1 and T2: I, $6.2 \pm 3.3, p=0.45, t(750)=0.75^{\mathrm{m}} ; \mathrm{YA}, 4.78 \pm 5, p=$ $0.52, t(408)=0.64^{\mathrm{n}} ; \mathrm{OA}, 13.83 \pm 2.71, p=0.011, t(383)$ $=2.55^{\circ}$; paired $t$ test; Fig. $3 D, E$ ).

In immature rats, short periods of auditory stimulation readily modify frequency tuning in $A 1$, likely because of a disrupted $\mathrm{E} / \mathrm{l}$ balance following the onset of hearing (Dorrn et al., 2010). Additionally, considering that $\mathrm{GABA}_{\mathrm{A}^{-}}$ mediated inhibition regulates SSA (Duque et al., 2014), we hypothesized that transiently increasing inhibitory tone would improve adaptation in both the immature and aged A1. To test this possibility, we administered the shortacting $\mathrm{GABA}_{A}$ agonist midazolam during the presentation of the same repetitive stimulus. Given the different time scales between our adaptation (10 $\mathrm{min}$ ) and passive exposure (1-2 weeks) experiments, we decided to use midazolam as opposed to the long-acting $\mathrm{GABA}_{\mathrm{A}}$ agonist diazepam. Direct application of midazolam (1 $\mu \mathrm{g} / \&$ micro; at a rate of $0.5 \mu \mathrm{l} / \mathrm{min}$ ) to the cortex resulted on average in the progressive suppression of $A 1$ responses to repetitive tones in the immature and older groups (median slope of normalized response rate: $I,-0.13 \pm 0.02 \times 10^{-4}, p=1.1$ $\times 10^{-5}, z=-4.4^{\mathrm{p}}$, number of recorded cortical sites $=$ 346; OA, $-0.01 \pm 0.04 \times 10^{-4}, p=0.013, z=-2.46^{q}$, number of recorded cortical sites $=155$; Wilcoxon ranksum test; Fig. 3F, G and Fig. 3-1A). It also resulted in a significant decrease in response to the high-probability tone in these groups (change in normalized firing rate in response to the standard tone, T2 minus T1; I: $-0.12 \pm$ $0.04, p<0.001, t(720)=5.29^{r}$; OA: $-0.09 \pm 0.06, p=$ $0.03, t(345)=2.1^{\text {s; }} t$ test). The overall response to the standard-oddball stimulus remained constant from $\mathrm{T} 1$ to T2 for both groups (difference in mean area under the curve between T1 and T2: I, $6.08 \pm 4.55, p=0.39, t(690)$ $=0.86^{\mathrm{t}}$; OA, $1.63 \pm 5.52, p=0.94, t(308)=0.08^{\mathrm{u}} ; t$ test; Fig. $3 H, I)$. A summary of $A 1$ responses to repetitive tones and oddballs is provided in Fig. 3-1B.

\section{Impact of aging and dysregulated plasticity on auditory learning}

Our results using passive sound exposure over different time scales suggest that age-related loss of inhibition could return the cortex into a state of instability where sensory representations are continuously distorted by nonspecific passive experience. If the deleterious effects of age-related loss of inhibition observed on passive experience extend to goal-oriented behavior, it is conceivable that reduced inhibition might contribute to make learning slower, harder, and more susceptible to decay, as has been clinically observed in older patients (Boyke et al., 2008; Lustig et al., 2009). To examine the impact of age on the retention of training-related plastic changes in A1, we compared the performance of young $(n=8)$ and older $(n=12)$ adult rats on an auditory discrimination task and then measured training-induced $\mathrm{A} 1$ changes at the end of training and after a 4-week delay. Both groups were trained on a two-tone discrimination task (target tone: $5 \mathrm{kHz}$, non-target tone: $10 \mathrm{kHz}$ ). Training ended once the rats' discrimination reached a sustained value of D-prime $\left(d^{\prime}\right) \geq 1$ for two consecutive days; Fig. $4 A$, top). Older rats required on average more training sessions to reach criterion than younger adults (YA: $8.4 \pm 0.8$ sessions; OA: $11.9 \pm 1.1, p=0.032, t(18)=2.32^{v}$; $t$ test; Fig. $4 A$, bottom). At the end of training, A1 CF maps were obtained from a subgroup of young (YA-T, $n=4$ ) and a subgroup of old adult rats (OA-T, $n=4$; Fig. 4B). Two-way analysis of variance revealed a significant effect of training $\times$ frequency bin for both YA-T and OA-T groups $(F(11,72)$ $=13.42, p<0.001^{\mathrm{w}} ; F(11,72)=6.57, p<0.001^{\mathrm{x}}$; respectively). Compared to age-matched controls, both groups exhibited an increase in the number of neurons tuned to the target tone by the end of the training period (average \% difference in the proportion of recording sites tuned within octave of exposure tone, relative to control: YA-T: $12.25 \pm 1.5 \%$ increase, $p=0.029^{\mathrm{w}}$; OA-T: $8.06 \pm$ $2.25 \%$ increase, $p=0.004^{\times}$, corrected with TukeyKramer test; Fig. 4C). We also found, as previously reported (Voss et al., 2016), that the nontarget frequency was underrepresented in the trained YA but not in the OA group (YA-T: $11.54 \pm 3.5 \%$ decrease, $p=0.018^{\mathrm{w}}$; OA-T: $6.7 \pm 2.2 \%$ decrease, $p=0.41^{\times}$, corrected with TukeyKramer test; Fig. 4C).

To determine the retention of learning and persistence of training-related $A 1$ retuning, we characterized trained younger $\left(\mathrm{YA}-\mathrm{T}_{\text {delay }}, n=4\right)$ and older $\left(\mathrm{OA}-\mathrm{T}_{\text {delay }}, n=4\right)$ rats after a 4-week delay period following completion of training (Fig. $4 D$, top). On average, younger rats maintained a significantly better performance than older when resuming training $\left(Y A-T_{\text {delay }}: d^{\prime}=2.8 \pm 0.12 ; O A-T_{\text {delay }}: d^{\prime}=\right.$ $0.96 \pm 0.48, p=0.002 t(6)=5.02^{y}$; Fig. $4 D$, bottom). A1 $\mathrm{CF}$ maps were reconstructed in another group of younger and older rats after the delay period (Fig. $4 E$ ). In these we found that the target tone representation in $\mathrm{A} 1$ had persisted in the younger but not older group (YA-T delay: $F(11,72)=6.68, p<0.001$, two-way ANOVA; $5 \mathrm{kHz}$ : $10.37 \pm 2.3 \%$ increase, $p=0.01^{z}$, corrected with TukeyKramer test; OA-T delay: $F(11,72)=1.41, p=0.18^{\mathrm{ab}}$, twoway ANOVA; $5 \mathrm{kHz}$ : $4.72 \pm 2.1 \%$ increase; Fig. $4 F$ ). Finally, to test whether pharmacologically increasing GABA inhibition would improve the retention of traininginduced plastic changes, we treated a subgroup $(n=4)$ of older rats with diazepam (1 mg/kg i.p., twice a day) during the delay period post-training. A1 mapping in this group revealed a persistent target tone overrepresentation not significantly different from what had been observed immediately following training $(F(11,72)=5.42, p<0.001$, two-way ANOVA; $5 \mathrm{kHz}: 7.64 \pm 2.8 \%$ increase, $p=$ $0.022^{\mathrm{ac}}$, corrected with Tukey-Kramer test; Fig. 4F). 
A
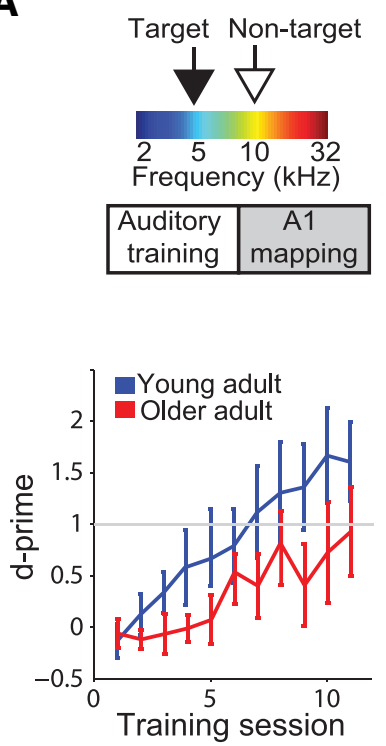

D
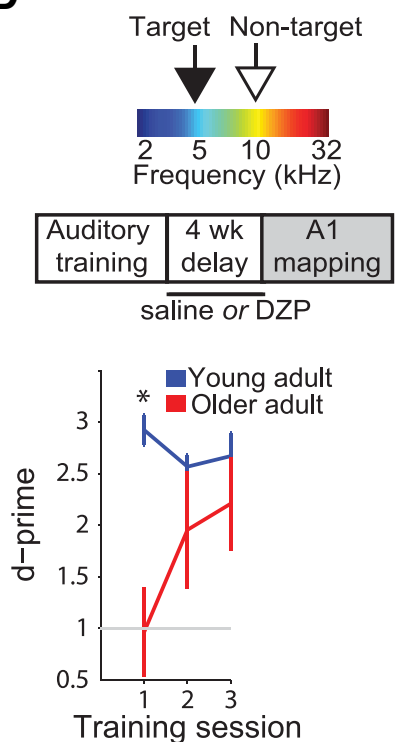

B Trained

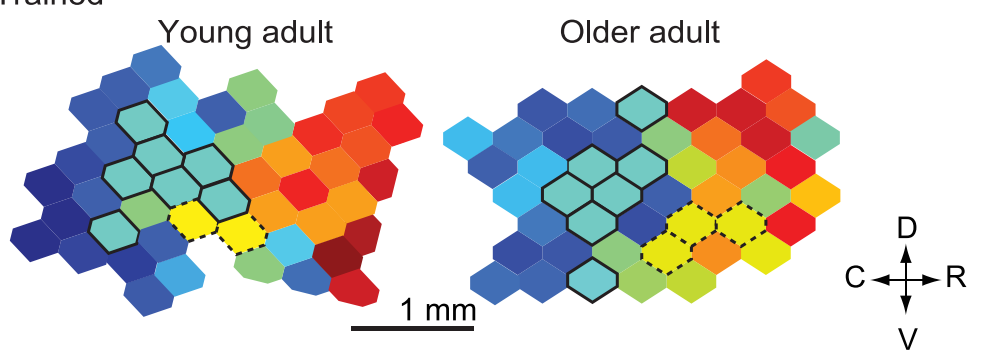

C

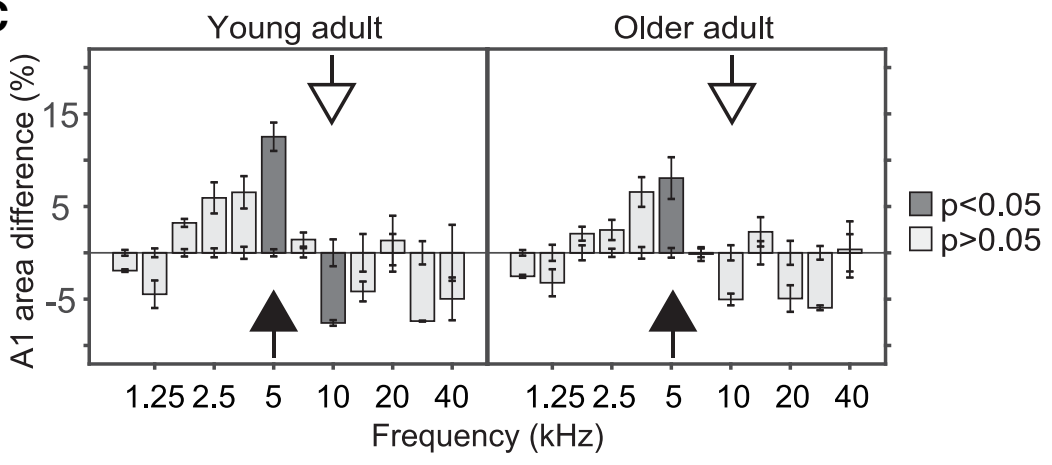

E Trained (delay)
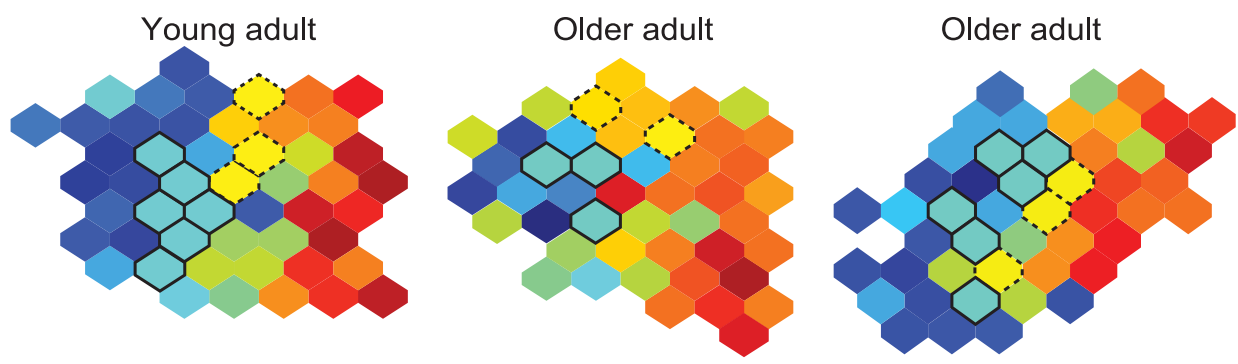

F

Young adult (delay)

Older adult (delay)

Older adult (delay)

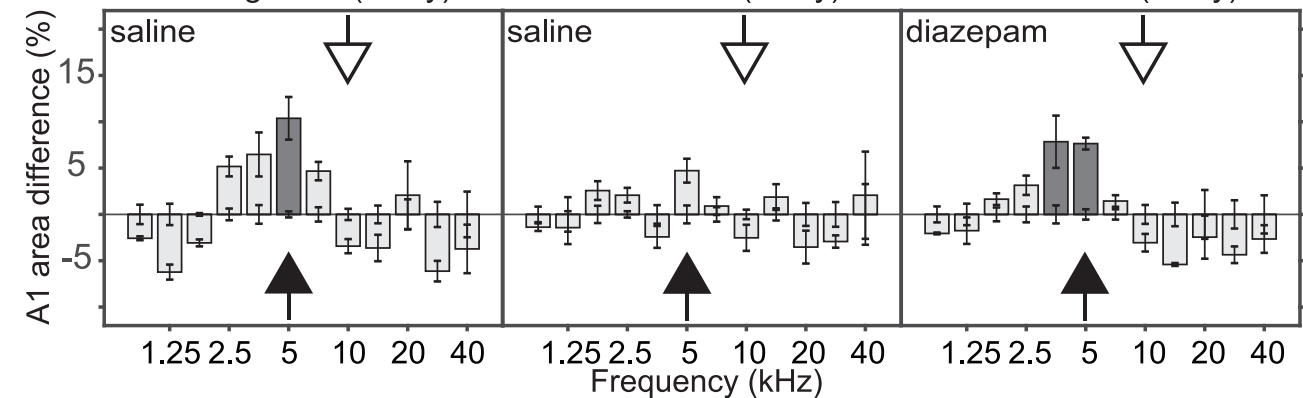

Figure 4. Aging and decay of training-induced $A 1$ plasticity. Young and old adult rats were trained on a two-tone discrimination task (target tone: $10 \mathrm{kHz}$, nontarget: $5 \mathrm{kHz}$ ). $\boldsymbol{A}$, Top: Experimental protocol. Bottom: Older adult rats needed on average more training sessions to reach criterion than young adult rats (D-prime $\geq 1$; YA no. of sessions $=8.4 \pm 1.1 ; \mathrm{OA}=11.9 \pm 1.4, p=0.03$ ). $\boldsymbol{B}$, Representative A1 characteristic frequency (CF) maps from trained young (left) and old (right) adult rats. Bolded polygons have a CF at the target tone \pm 0.3 octaves. Hatched polygons have a CF at the nontarget tone \pm 0.3 octaves. $\boldsymbol{C}$, Difference in frequency tuning between naïve and exposed rats expressed as A1 percentage area and separated by CF. The full arrows point to the target frequency; the hatched arrows points to the nontarget frequency. D, Top: To determine the persistence of learning and training-induced A1 map plasticity, a subgroup of YA-T and two subgroups of OA-T rats were subjected to a 4-week delay after reaching criterion, followed by behavioral re-assessment and A1 mapping. Bottom: From the first session of the reassessment onwards, young adult rats performed above criterion, while old adult rats performed above criterion from the second session onwards. $\boldsymbol{E}$, Representative A1 characteristic frequency (CF) maps from trained rats that received daily sham (saline) or diazepam (DZP) injections during the delay period. $\boldsymbol{F}$, Difference in A1 area tuned to various frequencies between each experimental group and untrained age-matched controls. YA-T group: $n=4$, recorded sites $=212$; OA-T: $n=4$, recorded sites $=209 ; Y_{\text {YA-Tay }}: n=4$; recorded sites $=192 ;$ OA-T delay $: n$ $=4$; recorded sites $=203 ;$ OA-T delay(DZP) $: n=4$; recorded sites $=189$. Values shown are mean \pm SEM, $t$ test, two-way ANOVA with Tukey-Kramer correction. 


\section{Tonic GABAergic inhibition is reduced in the aged A1}

To study the anatomic correlates of frequency tuning instability and impaired training performance, we sampled GABA concentration using microdialysis and quantified PV/PNN expression in A1 through immunohistochemistry. As documented in previous research (Morrison and Baxter, 2012; Rozycka and Liguz-Lecznar, 2017), we found that GABA concentration in A1 interstitial fluid was $25 \%$ lower in older adult rats $(\mathrm{OA}, 24$ months old, $n=4)$ than in young adult controls (YA, 6 months old, $n=4$ ) when measured in silence (YA, $100 \pm 7.9 \%$; OA, $75.2 \pm 5.8 \%$ relative to $Y A ; p=0.04, t(6)=2.53^{\text {ad }}, t$ test; Fig. $5-1 A$ ). This difference was more pronounced during continuous sound presentation (see Methods). In the latter experimental condition, a relative reduction close to $40 \%$ was noted (YA-stim, $124 \pm 7.7 \%$; OA-stim, $88.2 \pm 6.0 \%$ relative to YA-stim; $p=0.01, t(6)=3.66^{\mathrm{ae}}, t$ test; Fig. 5-1B).

\section{Impact of age on perineuronal nets and $\mathrm{PV}^{+}$neurons in $\mathrm{A} 1$}

PV- and SST-positive cells constitute the two largest interneuron subpopulations throughout the cortex. In particular, $\mathrm{PV}^{+}$neurons and associated PNNs are important regulators of experience-dependent plasticity throughout life (Caroni et al., 2012; Wang and Fawcett, 2012). Reduced cortical staining of PV and PNN are both associated with cortical immaturity and increased instability of cortical representations (Pizzorusso et al., 2002; McRae et al., 2007; Wang and Fawcett, 2012; Donato et al., 2013).

To assess whether age-related representational instability would be paralleled by a reduction in these plasticity-regulating structural elements, we first characterized the expression of the main interneuron subpopulations in the context of total cell counts for the three age groups-immature, young adult, and older adult-included in the present study (see Fig. 5-2 and Fig. 5-3 for cell counts and representative micrographs of interneurons, respectively). This analysis confirmed previous research showing a decrease in $\mathrm{PV}^{+}$and $\mathrm{SST}^{+}$cell counts associated with aging (Ouda et al., 2008; Ouellet and de Villers-Sidani, 2014). We then examined PV and PNN staining intensity in our different experimental groups (I, $n$ $=6$; YA, $n=6$; OA, $n=6$; ID, $n=3$; OAD, $n=3$; Fig. 5A). In line with previous reports (Hilbig et al., 2002; Ouda et al., 2008), we found decreased PV staining intensity with aging, which was recovered with 2-week-long diazepam treatment [median staining intensity \pm MAD per $\mathrm{PV}^{+}$cell, arbitrary confocal units $(\mathrm{au}) \times 10^{5} ; H(4)=14.52$, $p=0.0058$, Kruskal-Wallis test; I: $1.17 \pm 0.85, p=0.52$, relative to YA; YA: $1.19 \pm 0.69$; OA: $1 \pm 0.53, p=0.011$, relative to YA; ID: $1.3 \pm 0.66, p=0.96$, relative to $Y A$; OAD: $1.1 \pm 0.76, p=0.97^{\text {af }}$, relative to YA; Tukey-Kramer test; Fig. $5 B$ and Fig. 5-4A]. PNN staining intensity, in contrast, showed a more contrasting lifetime trajectory, increasing from immature to young adult age and then reversing course with aging (Fig. 5D). Like our findings on $\mathrm{PV}^{+}$cells, diazepam treatment resulted in recovery of
PNN intensity staining for immature and older adult rats (median staining intensity per PNN, au $\times 10^{5} ; H(4)=$ 83.97, $p<0.0001$, Kruskal-Wallis test; I: $0.7 \pm 0.79, p<$ 0.0001 , relative to YA; YA: $1.64 \pm 1.37$; OA: $0.76 \pm 1.04$, $p<0.001$, relative to YA; ID: $1.79 \pm 1.39, p=0.96$, relative to YA; OAD: $1.11 \pm 1.33, p=0.003^{\text {ag }}$, relative to YA; Tukey-Kramer test; Fig. $5 D$ and Fig. 5-4C).

Further examination revealed that staining intensity of individual $\mathrm{PV}^{+}$cells could be divided into four subgroups: low, intermediate low, intermediate high, and high intensity (Donato et al., 2013). We found a smaller proportion of high-intensity $\mathrm{PV}^{+}$cells in older rats compared to young adults $\left(H(4)=13, p=0.011^{\text {aj }}\right.$, Kruskal-Wallis test; fraction of $\mathrm{PV}^{+}$cells with low staining intensity and $p$-value relative to $Y A$, per group: $I_{\text {low }}=16 \pm 7.1 \%, p=0.82 ; \mathrm{YA}_{\text {low }}=14$ $\pm 3.2 \% ; \mathrm{OA}_{\text {low }}=2.9 \pm 0.4 \%, p=0.005 ; \mathrm{ID}_{\text {low }}=12.1 \pm$ $1.7 \%, p=0.99 ; \mathrm{OAD}_{\text {low }}=14.2 \pm 3.2 \%, p=0.99^{\text {ah }}$; Tukey-Kramer test; Fig. $5 C$ and Fig. 5-4B). A similar analysis was performed on PNNs, which could also be divided into four staining intensity groups. We found on average a higher proportion of low-intensity PNNs in aged rats compared to young adults $(H(4)=17.24, p=0.0017$, KruskalWallis test; fraction of low-intensity PNNs and $p$-value relative to $Y A$, per group: $\mathrm{I}_{\text {low }}=55 \pm 8.1 \%, p=0.48$; $\mathrm{YA}_{\text {low }}=34 \pm 2.2 \%$; $\mathrm{OA}_{\text {low }}=55 \pm 1.7 \%, p=0.04^{\mathrm{ai}}$, Tukey-Kramer test) and a decrease in the high-intensity PNN subgroup in immature and aged rats compared to young adults $(H(4)=22.06, p<0.001$, Kruskal-Wallis test; fraction of high-intensity PNNs and $p$-value relative to $Y A$, per group: $I_{\text {high }}=3.5 \pm 1.7 \%, p=0.004 ; Y_{A_{\text {high }}}=$ $26 \pm 1.9 \% ; \mathrm{OA}_{\text {high }}=9.3 \pm 2.7 \%, p=0.039^{\text {aj; }}$ TukeyKramer test). Notably, following diazepam treatment, the proportion of low-intensity PNNs in older rats decreased, whereas the proportion of high-intensity PNNs in both immature and older rats increased, resulting in an intensity staining distribution that resembled that of the control (YA) group (fraction of PNN as a function of staining intensity and $p$-value relative to $Y A$, per group: $I_{\text {low }}=$ $31.1 \pm 1.9 \%, p=0.8 ; \mathrm{OAD}_{\text {low }}=47 \pm 3.1 \%, p=0.99^{\text {ai. }}$; $I D_{\text {high }}=27.2 \pm 0.5 \%, p=0.99 ; \mathrm{OAD}_{\text {high }}=21.2 \pm 3.5 \%$, $p=0.85^{\mathrm{aj}}$; Tukey-Kramer test; Fig. $5 E$ and Fig. 5-4D).

\section{Discussion}

Our findings indicate that experience-dependent plasticity increases with aging following a natural reduction in cortical inhibition. Such increased plasticity may facilitate changes elicited by experience but also impair the brain's capacity to crystallize such changes.

Brain aging is characterized by a down-regulation of cortical inhibition, which contributes to a range of functional deficits such as reduced selectivity of receptive fields, degraded temporal processing, heightened responses to noise, and reduced adaptation to repetitive stimuli (Turner et al., 2005; Hua et al., 2006; Caspary et al., 2008; Liguz-Lecznar et al., 2014; Schreiner and Polley, 2014).

What are the mechanisms of age-related reduction in inhibition? Recent findings suggest that reduced inhibition might not be a result of aging itself (Gourevitch et al., 
A
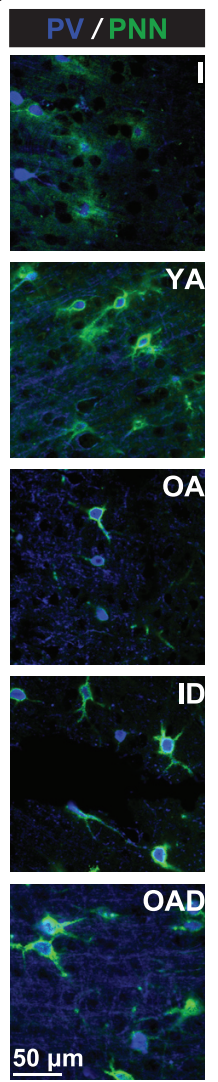

B
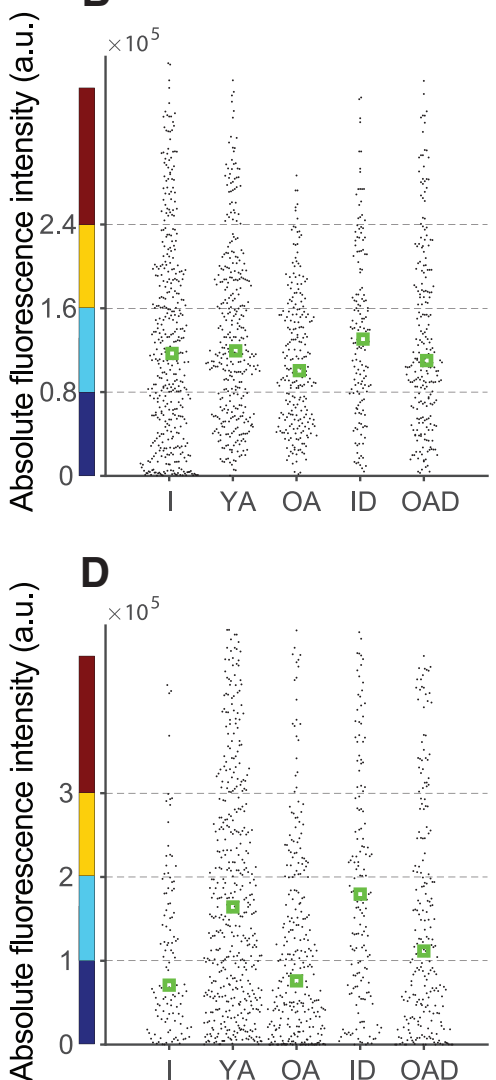
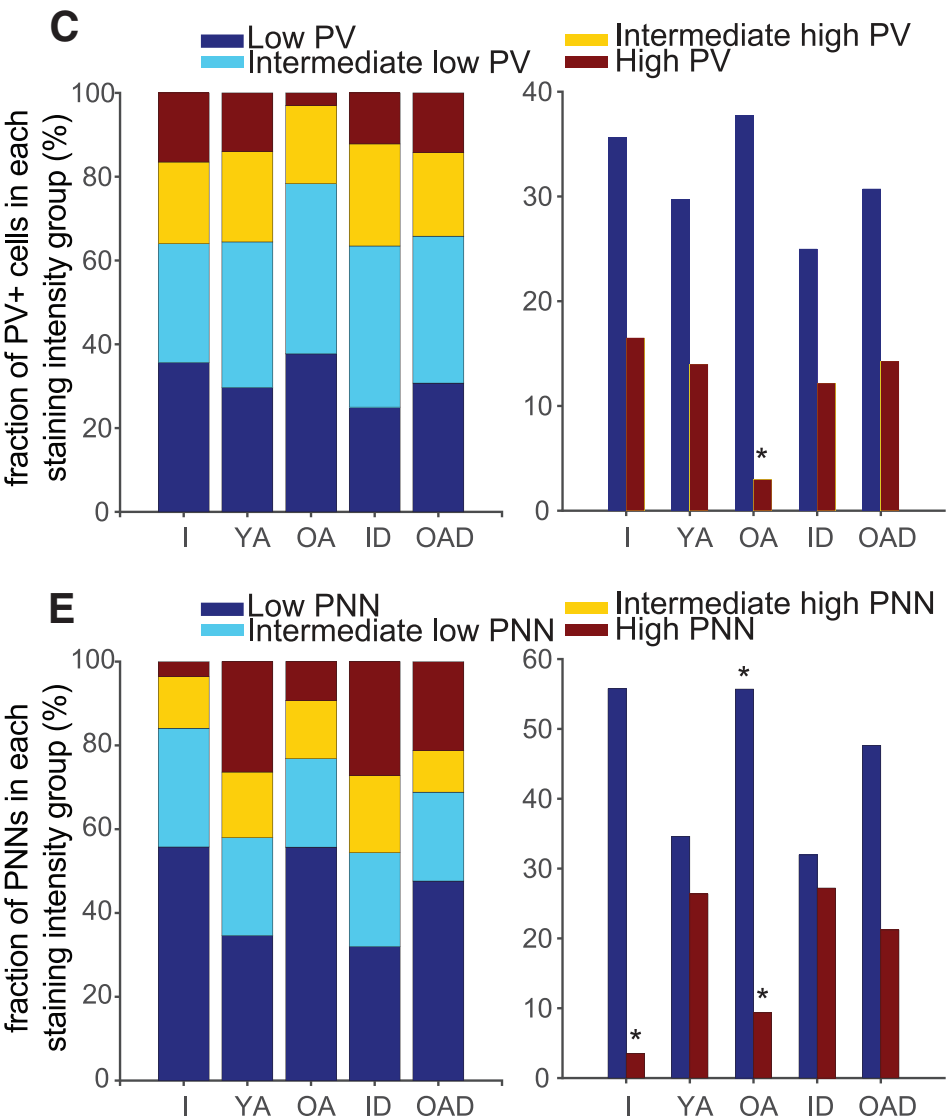

Intermediate high PNN

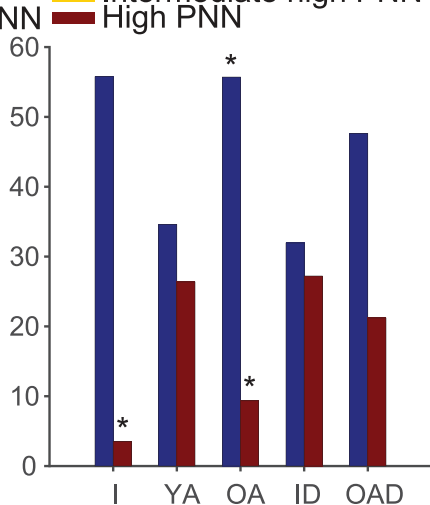

Figure 5. Impact of age on structural inhibitory elements in the auditory cortex. A, High-power microphotographs of representative sections immunolabeled for perineuronal nets (PNN) and parvalbumin (PV) from immature (I), young adult (YA), old adult, immature + diazepam treatment (IA), and old adult + diazepam treatment (OAD) rats. $\boldsymbol{B}, \boldsymbol{D}$, Group fluorescence optical density for (B) PV and (D) PNN staining for each age group (all cortical layers; green boxes represent median values). $\boldsymbol{C}, \boldsymbol{E}$, Distribution of (C) PV cell and $(E)$ PNN intensity staining for each age group. Fig. 5-1 compares A1 GABA concentration between YA and OA rats. Cell count per field for different neuronal types and age groups are detailed in Fig. 5-2. Fig. 5-3 shows representative micrographs of PV- and SST-positive cells. A summary of the cumulative distribution of staining intensity and interindividual variability for all groups is provided in Fig. 5-4. Number of hemispheres examined: I =12, YA =12, OA =12, ID =6, OAD = 6; total cell count per group: I = $418, \mathrm{YA}=343, \mathrm{OA}=236, \mathrm{ID}=156, \mathrm{OAD}=231$. Values shown are mean \pm SEM. $* p<0.05$ relative to YA; Kruskal-Wallis test, corrected for multiple comparisons using Tukey-Kramer test.

2014). Young rats housed in a noisy auditory environment exhibit auditory perceptual deficits that mirror those observed in aging (Kamal et al., 2013; Gourevitch et al., 2014), alongside reduced GABA and interneuron expression (Zhou et al., 2011; Zhou and Merzenich, 2012). These impairments, however, are observed exclusively in rats exposed to continuous nonmodulated noise, but not after amplitude-modulated noise exposure (Thomas et al., 2018), suggesting that it is the lack of structured inputs - as opposed to noise per se-that drives maladaptive plasticity in the auditory cortex (Voss et al., 2017). It is therefore possible that age-related maladaptive plastic changes are a consequence of continuous, nonstructured "noisy" inputs, whether originating from the environment or resulting from conductive, sensorineural, or strial hearing loss (Jayakody et al., 2018). Prolonged exposure to distorted inputs might destabilize the activity of local neural circuits (Gourevitch et al., 2014) and trigger compensatory homeostatic changes (Burrone and Murthy, 2003; Dean et al., 2005; Turrigiano, 2011) that ultimately amplify excitatory inputs and reduce inhibition (Rothman et al., 2009; Tyagarajan et al., 2011).

The aforementioned studies strongly suggest that agerelated anatomic and functional deficits can be modeled in noise-exposed young adult rats. Furthermore, rats exposed to nonstructured noise recover normal function when returned to their normal environment (Zhou and Merzenich, 2012; Kamal et al., 2013). Taken together, these observations suggest that perceptual deficits observed in the aged cortex have a significant activitydependent component, rather than being purely agerelated, and are thus at least partially reversible (Hilbig et al., 2002; Zhou and Merzenich, 2012; Liguz-Lecznar et al., 2014). For instance, GABA agonists increase selectivity of receptive fields in the primary visual cortex (Leventhal et al., 2003; Hua et al., 2006), classic conditioning enhances the expression of GABAergic markers in the barrel cortex (Liguz-Lecznar et al., 2014), and operant conditioning results in increased PV expression in A1 (de Villers-Sidani et al., 2010). 


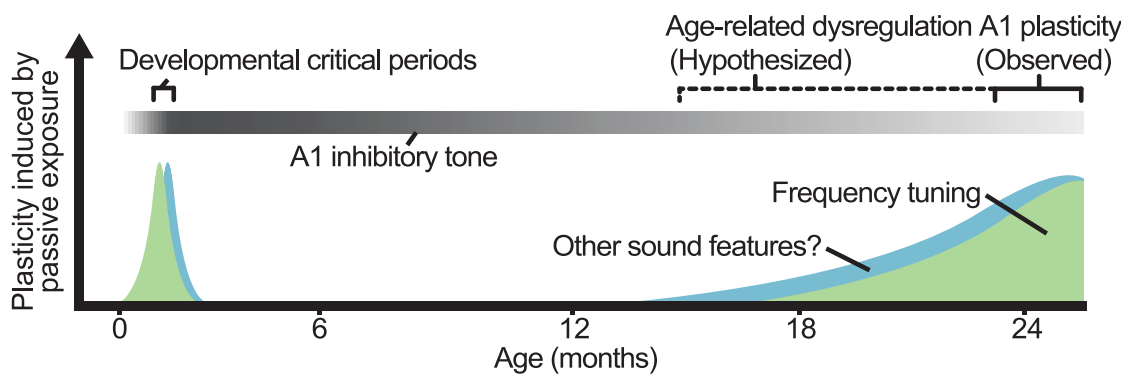

Figure 6. Proposed model of the impact of age on A1 plasticity. During periods of life characterized by a low inhibitory tone, passive exposure alters the A1 CF map. Plastic changes to the immature A1 are long lasting: as inhibition increases, the CP ends and sensory representations become stable. In contrast, plastic changes to the aged A1 are short-lived, as these cannot be consolidated due to a persistent low inhibitory tone.

Functional deficits in the aged A1 include slowed and incomplete suppression of background distractors, which further impairs the detection of novel stimuli (de VillersSidani et al., 2010; Mishra et al., 2014). In the present study, this deficit was evident on a 10-min-long exposure to repetitive tones (Fig. 3B-E). We found impaired adaptation and tuning instability in the aged $A 1$, whereas increasing inhibition with a short-acting GABAA agonist improved adaptation and reversed the tendency of aged A1 neurons to increase their tuning to the repetitive tone (Fig. 3F-l). Although there might be differences in the physiologic response to anesthesia between aged and adult animals, it should be noted that SSA is a property found in A1 and subcortical auditory nuclei that is minimally affected by anesthesia (Richardson et al., 2013; Duque and Malmierca, 2015).

Tuning instability was further confirmed by the fact that a short 1-week pip tone exposure sufficed to produce an overrepresentation of the exposure tone in older rats, as previously seen in immature rats (Fig. 1). However, this increased plasticity in the aged auditory cortex does not seem to be limited to a short time window, as is the case with the CP. In the present study, immature rats exposed successively to two different pure tones exhibited plasticity in response to the first tone, most likely because only the first tone exposure overlapped with the CP (Fig. 2A). Whereas a rapid and sustained increase in inhibition ( $\mathrm{Fa}$ giolini and Hensch, 2000; Iwai et al., 2003; Hensch, 2005) ends the CP and prevents additional alterations due to passive sound exposure, a subsequent 1-week exposure to a different tone resulted in the overrepresentation of the latter tone in aged rats. Interestingly, boosting GABA inhibition consolidated frequency tuning representation and made the aging $\mathrm{A} 1$ again resistant to further alterations, thus "closing" this period of maladaptive increased plasticity (Fig. 2C). Follow-up studies may want to rule out the possibility, although unlikely, that diazepam selectively affects the processing of frequency tones in the $10-\mathrm{kHz}$ range by presenting a lower frequency tone (e.g. 5 $\mathrm{kHz}$ ) before the $10-\mathrm{kHz}$ tone during diazepam treatment. Taken together, these findings suggest that the aging $A 1$ appears to be in a permanent state of heightened plasticity to levels akin to those observed during early development.
The slower rate of learning in aged rats supports previous findings showing that age-related cortical processing deficits contribute to degraded behavioral performance (Barnes et al., 1997; Gazzaley et al., 2005; Samson and Barnes, 2013; Fig. 4A-C). According to the map expansion-renormalization model, initial sensory map expansion is necessary for discrimination learning (Takahashi et al., 2010; Reed et al., 2011). However, once subjects become experts at a task and reach a plateau in performance, their maps return to their previous state (Reed et al., 2011). In the present study, training was suspended before rats reached this plateau, and both groups exhibited typical training-induced map changes (Blake et al., 2006; Polley et al., 2006; Zhou et al., 2010). Learning becomes more susceptible to decay with aging (Lustig et al., 2009), which was evident after a 1-month delay period (Fig. 4D-F). Interestingly, training-induced map changes were preserved in the old rats treated with diazepam during the delay period between end of training and cortical mapping. Although map expansion was still present after this relatively short delay period, we did not measure the behavioral implications or the extent of this persistence beyond 1-month follow-up. Further studies will be necessary to fully understand the behavioral relevance of sustained map plasticity for learning.

Our findings of reduced PV and SST expression support numerous reports of reduced interneuron cell counts associated with aging (Rozycka and Liguz-Lecznar, 2017), suggesting that inhibitory deficits may be related to the dysfunction of specific interneuron cell subtypes (Cha et al., 1997; Ouda et al., 2008; Fish et al., 2013). Recent research, however, has focused on PV expression as a proxy for cellular function and has shown that cortical PV staining intensity is tightly correlated with the degree of experience-dependent plasticity (Zhou et al., 2011; Caroni, 2015). As a case in point, recent studies by Donato et al. $(2013,2015)$ demonstrate the impact of reduced PV staining on cell function. High-intensity $\mathrm{PV}^{+}$cells are found on completion of learning and immediately after fear conditioning, situations in which stable, long-lasting sensory representations are warranted (Donato et al., 2013). Conversely, low-intensity $\mathrm{PV}^{+}$cells are abundant during learning and following environmental enrichment, situations in which a more flexible cortical network is 
needed. Similarly, interventions that delay cortical maturation during early development (Chang and Merzenich, 2003; de Villers-Sidani et al., 2008) and those that impair auditory processing during adulthood (Martin del Campo et al., 2012; Zhou and Merzenich, 2012) result in decreased PV staining and increased plasticity. In line with these observations, we found a moderate increase in the low-PV fraction in the immature and aged $A 1$, the age groups that showed increased experience-dependent plasticity. Moreover, the high-PV fraction was significantly diminished in the aged (Fig. 5B, C), which could account for the inadequate consolidation of newly formed sensory representations (Caroni et al., 2012; Donato et al., 2013).

PNNs are extracellular matrix deposits produced jointly by neurons and astrocytes, particularly around $\mathrm{PV}^{+}$cells (McRae et al., 2007; Nakamura et al., 2009), forming both a structural and functional barrier that limits plasticity (Pizzorusso et al., 2002; Berardi et al., 2004; Wang and Fawcett, 2012). We found age-related changes in PNNs that mirrored those documented for $\mathrm{PV}^{+}$cells; namely, a lower average staining density of PNNs in the extremes of life, characterized by an increase in the low-PNN fraction and a decrease in the high-PNN fraction. Interestingly, while age-related PNN intensity differences were more striking than those observed for $\mathrm{PV}^{+}$cells, diazepam treatment in both cases resulted in a redistribution of the low- and high-intensity subgroups in immature and older adult rats toward values that resembled those of the young adult group (Fig. 5D, E). The disparity in histology results between $\mathrm{PV}^{+}$cells and associated PNNs in immature rats supports the idea that $\mathrm{PV}^{+}$cell development predates PNN assembly (Baker et al., 2017), suggesting that adequate $\mathrm{PV}^{+}$cell functioning is required for PNN formation (Yamada et al., 2015; Quattromani et al., 2017).

The present study contributes to the understanding of how plasticity is regulated in the aged brain. Whereas previous studies have shown that GABAergic inhibition declines with age (Leventhal et al., 2003; Caspary et al., 2008; Liguz-Lecznar et al., 2014) and that passive sound exposure can alter cortical response properties in adulthood (Norena et al., 2006; Pienkowski et al., 2011), our study is the first one to show that A1 experience-dependent plasticity increases with aging. Further targeted manipulations of GABAergic function will be necessary to pinpoint the exact mechanisms underlying this age-related dysregulation of plasticity and to understand whether altered excitatory neurotransmission during aging (Benali et al., 2008) also plays a role.

Our findings have the potential to inform future research in animal models and humans. Recent studies have shown that cortical interneurons gate critical period plasticity locally (Takesian et al., 2018) and are necessary for sustained behavioral performance in trained animals (Kuchibhotla et al., 2017), but long-term outcomes of manipulating inhibitory neurotransmission remain unknown. Although we used a systemic GABA agonist, a logical next step in animal research would be to modulate inhibitory neurotransmission locally during passive exposure or learning using optogenetics or DREADDs for acute or chronic interventions, respectively.
In the human research domain, our findings may be particularly relevant to studies that are currently underway and that have potential clinical applications. In the absence of region-selective drugs to modulate GABAergic neurotransmission, studies using noninvasive brain stimulation (NIBS) are exploring the effects of manipulating cortical $E / /$ balance on learning in the elderly (Opie and Cirillo, 2017). For instance, Opie et al. (2017) used two modalities of transcranial magnetic stimulation to alter cortical excitability before a motor learning task but found no benefit in healthy aged volunteers. In contrast, a subsequent study using transcranial direct current stimulation found that increasing inhibition before testing, followed by decreasing inhibition during testing, resulted in greater skill improvement in older adults (Fujiyama et al., 2017). We posit that a follow-up experiment using NIBS could be used to test our hypotheses of the role of inhibition in the acquisition and retention of learning-specifically, to test whether reducing inhibition early during training increases plasticity and facilitates learning, and whether increasing inhibition after learning facilitates the crystallization of newly acquired skills.

Traditionally, aging has been regarded as a period of limited plasticity. However, our experiments suggest that this idea is unlikely to be correct in detail, as the aged brain is in some ways more plastic than the young adult brain. We propose that the inhibitory regulation of plasticity, rather than plasticity per se, is reduced in the aged brain (Fig. 6). Researchers and clinicians may build on this knowledge to develop rehabilitation strategies with at least two complementary objectives in mind: first, taking advantage of increased plasticity to enhance seniors' functional recovery after neurologic injury, and second, regulating plasticity to preserve the benefits of rehabilitation and promote long-lasting recovery.

\section{References}

Baker KD, Gray AR, Richardson R (2017) The development of perineuronal nets around parvalbumin gabaergic neurons in the medial prefrontal cortex and basolateral amygdala of rats. Behav Neurosci 131:289-303. CrossRef

Barnes CA, Suster MS, Shen J, Mcnaughton BL (1997) Multistability of cognitive maps in the hippocampus of old rats. Nature 388:272275. CrossRef

Benali A, Weiler E, Benali Y, Dinse HR, Eysel UT (2008) Excitation and inhibition jointly regulate cortical reorganization in adult rats. $J$ Neurosci 28:12284-12293. CrossRef Medline

Berardi N, Pizzorusso T, Maffei L (2004) Extracellular matrix and visual cortical plasticity: freeing the synapse. Neuron 44:905-908. CrossRef

Blake DT, Heiser MA, Caywood M, Merzenich MM (2006) Experience-dependent adult cortical plasticity requires cognitive association between sensation and reward. Neuron 52:371-381. CrossRef

Boyke J, Driemeyer J, Gaser C, Buchel C, May A (2008) Traininginduced brain structure changes in the elderly. J Neurosci 28: 7031-7035. CrossRef

Burrone J, Murthy VN (2003) Synaptic gain control and homeostasis. Curr Opin Neurobiol 13:560-567. Medline

Caroni P, Donato F, Muller D (2012) Structural plasticity upon learning: regulation and functions. Nat Rev Neurosci 13:478-490. CrossRef Medline 
Caroni P (2015) Regulation of Parvalbumin Basket cell plasticity in rule learning. Biochem Biophys Res Commun 460:100-103. CrossRef Medline

Carulli D, Pizzorusso T, Kwok JC, Putignano E, Poli A, Forostyak S, Andrews MR, Deepa SS, Glant TT, Fawcett JW (2010) Animals lacking link protein have attenuated perineuronal nets and persistent plasticity. Brain 133:2331-2347. CrossRef

Caspary DM, Ling L, Turner JG, Hughes LF (2008) Inhibitory neurotransmission, plasticity and aging in the mammalian central auditory system. J Exp Biol 211:1781-1791. CrossRef

Cha Cl, Lee YI, Lee EY, Park KH, Baik SH (1997) Age-related changes of VIP, NPY and somatostatin-immunoreactive neurons in the cerebral cortex of aged rats. Brain Res 753:235-44. CrossRef

Chang EF, Merzenich MM (2003) Environmental noise retards auditory cortical development. Science 300:498-502. CrossRef

de Villers-Sidani E, Chang EF, Bao S, Merzenich MM (2007) Critical period window for spectral tuning defined in the primary auditory cortex (A1) in the rat. J Neurosci 27:180-189. CrossRef

de Villers-Sidani E, Simpson KL, Lu YF, Lin RC, Merzenich MM (2008) Manipulating critical period closure across different sectors of the primary auditory cortex. Nat Neurosci 11:957-965. CrossRef

de Villers-Sidani E, Alzghoul L, Zhou X, Simpson KL, Lin RC, Merzenich MM (2010) Recovery of functional and structural agerelated changes in the rat primary auditory cortex with operant training. Proc Natl Acad Sci U S A 107:13900-13905. CrossRef

Dean I, Harper NS, Mcalpine D (2005) Neural population coding of sound level adapts to stimulus statistics. Nat Neurosci 8:16841689. CrossRef Medline

Donato F, Rompani SB, Caroni P (2013) Parvalbumin-expressing basket-cell network plasticity induced by experience regulates adult learning. Nature 504:272-276. CrossRef

Donato F, Chowdhury A, Lahr M, Caroni P (2015) Early- and lateborn parvalbumin basket cell subpopulations exhibiting distinct regulation and roles in learning. Neuron 85:770-786. CrossRef

Dorrn AL, Yuan K, Barker AJ, Schreiner CE, Froemke RC (2010) Developmental sensory experience balances cortical excitation and inhibition. Nature 465:932-936. CrossRef

Duque D, Malmierca MS, Caspary DM (2014) Modulation of stimulus-specific adaptation by GABA(A) receptor activation or blockade in the medial geniculate body of the anaesthetized rat. $J$ Physiol 592:729-743. CrossRef Medline

Duque D, Malmierca MS (2015) Stimulus-specific adaptation in the inferior colliculus of the mouse: anesthesia and spontaneous activity effects. Brain Struct Funct 220:3385-3398. CrossRef

Fagiolini M, Hensch TK (2000) Inhibitory threshold for critical-period activation in primary visual cortex. Nature 404:183-186. CrossRef Medline

Fish KN, Hoftman GD, Sheikh W, Kitchens M, Lewis DA (2013) Parvalbumin-containing chandelier and basket cell boutons have distinctive modes of maturation in monkey prefrontal cortex. J Neurosci 33:8352-8358. CrossRef

Fritschy JM, Panzanelli $P$ (2014) GABAA receptors and plasticity of inhibitory neurotransmission in the central nervous system. Eur $\mathrm{J}$ Neurosci 39:1845-1865. CrossRef

Froemke RC, Carcea I, Barker AJ, Yuan K, Seybold BA, Martins AR, Zaika N, Bernstein H, Wachs M, Levis PA, Polley DB, Merzenich MM, Schreiner CE (2013) Long-term modification of cortical synapses improves sensory perception. Nat Neurosci 16:79-88. CrossRef

Fujiyama $\mathrm{H}$, Hinder MR, Barzideh A, VAN DE Vijver C, Badache AC, Manrique CM, Reissig P, Zhang X, Levin O, Summers JJ, Swinnen SP (2017) Preconditioning tDCS facilitates subsequent tDCS effect on skill acquisition in older adults. Neurobiol Aging 51:31-42. CrossRef

Gazzaley A, Cooney JW, Rissman J, D’Esposito M (2005) Top-down suppression deficit underlies working memory impairment in normal aging. Nat Neurosci 8:1298-1300. CrossRef

Gourevitch B, Edeline JM, Occelli F, Eggermont JJ (2014) Is the din really harmless? Long-term effects of non-traumatic noise on the adult auditory system. Nat Rev Neurosci 15:483-491. CrossRef Medline

Hensch TK, Fagiolini M, Mataga N, Stryker MP, Baekkeskov S, Kash SF (1998) Local GABA circuit control of experience-dependent plasticity in developing visual cortex. Science 282:1504-1508. CrossRef

Hensch TK (2005) Critical period plasticity in local cortical circuits. Nat Rev Neurosci 6:877-888. CrossRef Medline

Hilbig H, Bidmon HJ, Steingruber S, Reinke H, Dinse HR (2002) Enriched environmental conditions reverse age-dependent gliosis and losses of neurofilaments and extracellular matrix components but do not alter lipofuscin accumulation in the hindlimb area of the aging rat brain. J Chem Neuroanat 23:199-209. CrossRef

Hua T, Li X, He L, Zhou Y, Wang Y, Leventhal AG (2006) Functional degradation of visual cortical cells in old cats. Neurobiol Aging 27:155-162. CrossRef

Iwai Y, Fagiolini M, Obata K, Hensch TK (2003) Rapid critical period induction by tonic inhibition in visual cortex. J Neurosci 23:66956702. Medline

Jayakody DMP, Friedland PL, Martins RN, Sohrabi HR (2018) Impact of aging on the auditory system and related cognitive functions: a narrative review. Front Neurosci 12:125. CrossRef

Kamal B, Holman C, DE Villers-Sidani E (2013) Shaping the aging brain: role of auditory input patterns in the emergence of auditory cortical impairments. Front Syst Neurosci 7:52. CrossRef

Knoflach M, Matosevic B, Rucker M, Furtner M, Mair A, Wille G, Zangerle A, Werner P, Ferrari J, Schmidauer C, Seyfang L, Kiechl S, Willeit J (2012) Functional recovery after ischemic stroke-a matter of age: data from the Austrian Stroke Unit Registry. Neurology 78:279-285. CrossRef

Kuchibhotla KV, Gill JV, Lindsay GW, Papadoyannis ES, Field RE, Sten TA, Miller KD, Froemke RC (2017) Parallel processing by cortical inhibition enables context-dependent behavior. Nat Neurosci 20:62-71. CrossRef

Kuhlman SJ, Olivas ND, Tring E, Ikrar T, Xu X, Trachtenberg JT (2013) A disinhibitory microcircuit initiates critical-period plasticity in the visual cortex. Nature 501:543-546. CrossRef

Lehmann K, Steinecke A, Bolz J (2012) GABA through the ages: regulation of cortical function and plasticity by inhibitory interneurons. Neural Plast 2012:892784. CrossRef Medline

Leventhal AG, Wang Y, PU, M, Zhou Y, MA, Y (2003) GABA and its agonists improved visual cortical function in senescent monkeys. Science 300:812-815. CrossRef

Liguz-Lecznar M, Lehner M, Kaliszewska A, Zakrzewska R, Sobolewska A, Kossut M (2014) Altered glutamate/GABA equilibrium in aged mice cortex influences cortical plasticity. Brain Struct Funct 220:1681-1693.

Lustig C, Shah P, Seidler R, Reuter-Lorenz PA (2009) Aging, training, and the brain: a review and future directions. Neuropsychol Rev 19:504-522. CrossRef Medline

Maffei A, Turrigiano G (2008) The age of plasticity: developmental regulation of synaptic plasticity in neocortical microcircuits. Prog Brain Res 169:211-223.

Malmierca MS, Sanchez-Vives MV, Escera C, Bendixen A (2014) Neuronal adaptation, novelty detection and regularity encoding in audition. Front Syst Neurosci 8:111. CrossRef

Martin DEL Campo HN, Measor KR, Razak KA (2012) Parvalbumin immunoreactivity in the auditory cortex of a mouse model of presbycusis. Hear Res 294:31-39. CrossRef Medline

Mcrae PA, Rocco MM, Kelly G, Brumberg JC, Matthews RT (2007) Sensory deprivation alters aggrecan and perineuronal net expression in the mouse barrel cortex. J Neurosci 27:5405-5413. CrossRef

Mishra J, DE, Villers-Sidani E, Merzenich M, Gazzaley A (2014) Adaptive training diminishes distractibility in aging across species. Neuron 84:1091-1103. CrossRef

Morrison JH, Baxter MG (2012, Mar 7) The aging cortical synapse: hallmarks and implications for cognitive decline. Nat Rev Neurosci 13(4):240-250. CrossRef Medline 
Nakamura M, Nakano K, Morita S, Nakashima T, Oohira A, Miyata S (2009) Expression of chondroitin sulfate proteoglycans in barrel field of mouse and rat somatosensory cortex. Brain Res 1252:117129. CrossRef

Norena AJ, Gourevitch B, Aizawa N, Eggermont JJ (2006) Spectrally enhanced acoustic environment disrupts frequency representation in cat auditory cortex. Nat Neurosci 9:932-939. CrossRef

Opie GM, Cirillo J (2017) Commentary: Preconditioning tDCS facilitates subsequent tDCS effect on skill acquisition in older adults. Front Aging Neurosci 9:84. CrossRef

Opie GM, Vosnakis E, Ridding MC, Ziemann U, Semmler JG (2017) Priming theta burst stimulation enhances motor cortex plasticity in young but not old adults. Brain Stimul 10:298-304.

Ouda L, Druga R, Syka J (2008) Changes in parvalbumin immunoreactivity with aging in the central auditory system of the rat. Exp Gerontol 43:782-789. CrossRef Medline

Ouellet L, DE Villers-Sidani E (2014) Trajectory of the main GABAergic interneuron populations from early development to old age in the rat primary auditory cortex. Front Neuroanat 8:40. CrossRef

Paxinos G, Watson C, 2007. The rat brain in stereotaxic coordinates. Amsterdam; Boston: Academic Press/Elsevier.

Pienkowski M, Munguia R, Eggermont JJ (2011) Passive exposure of adult cats to bandlimited tone pip ensembles or noise leads to long-term response suppression in auditory cortex. Hear Res 277: 117-126. CrossRef Medline

Pizzorusso T, Medini P, Berardi N, Chierzi S, Fawcett JW, Maffei L (2002) Reactivation of ocular dominance plasticity in the adult visual cortex. Science 298:1248-1251. CrossRef Medline

Polley DB, Steinberg EE, Merzenich MM (2006) Perceptual learning directs auditory cortical map reorganization through top-down influences. J Neurosci 26:4970-4982. CrossRef

Quattromani MJ, Pruvost M, Guerreiro C, Backlund F, Englund E, Aspberg A, Jaworski T, Hakon J, Ruscher K, Kaczmarek L, Vivien D, Wieloch T (2017) Extracellular matrix modulation is driven by experience-dependent plasticity during stroke recovery. Mol Neurobiol 55:2196-2213.

Reed A, Riley J, Carraway R, Carrasco A, Perez C, Jakkamsetti V, Kilgard MP (2011) Cortical map plasticity improves learning but is not necessary for improved performance. Neuron 70:121-131. CrossRef

Reinhoud NJ, Brouwer HJ, VAN Heerwaarden LM, Korte-Bouws GA (2013) Analysis of glutamate, GABA, noradrenaline, dopamine, serotonin, and metabolites using microbore UHPLC with electrochemical detection. ACS Chem Neurosci 4:888-894. CrossRef

Richardson BD, Hancock KE, Caspary DM (2013) Stimulus-specific adaptation in auditory thalamus of young and aged awake rats. $J$ Neurophysiol 110:1892-1902. CrossRef Medline

Rothman JS, Cathala L, Steuber V, Silver RA (2009) Synaptic depression enables neuronal gain control. Nature 457:1015-1018. CrossRef Medline

Rozycka A, Liguz-Lecznar M (2017) The space where aging acts: focus on the GABAergic synapse. Aging Cell 16:634-643. CrossRef Medline

Samson RD, Barnes CA (2013) Impact of aging brain circuits on cognition. Eur J Neurosci 37:1903-1915. CrossRef Medline
Schreiner CE, Polley DB (2014) Auditory map plasticity: diversity in causes and consequences. Curr Opin Neurobiol 24:143-156. CrossRef Medline

Stanley EM, Fadel JR, Mott DD (2012) Interneuron loss reduces dendritic inhibition and GABA release in hippocampus of aged rats. Neurobiol Aging 33:431.e1-e13. CrossRef

Stebbings KA, Choi HW, Ravindra A, Caspary DM, Turner JG, Llano DA (2016) Ageing-related changes in GABAergic inhibition in mouse auditory cortex, measured using in vitro flavoprotein autofluorescence imaging. J Physiol 594:207-221. CrossRef

Takahashi H, Funamizu A, Mitsumori Y, Kose H, Kanzaki R (2010) Progressive plasticity of auditory cortex during appetitive operant conditioning. Biosystems 101:37-41. CrossRef

Takesian AE, Bogart LJ, Lichtman JW, Hensch TK (2018) Inhibitory circuit gating of auditory critical-period plasticity. Nat Neurosci 21:218-227. CrossRef

Thomas ME, Friedman NHM, Cisneros-Franco JM, Ouellet L, DE, Villers-Sidani E (2018) The prolonged masking of temporal acoustic inputs with noise drives plasticity in the adult rat auditory cortex. Cereb Cortex

Turner JG, Hughes LF, Caspary DM (2005) Affects of aging on receptive fields in rat primary auditory cortex layer $\mathrm{V}$ neurons. J Neurophysiol 94:2738-2747. CrossRef Medline

Turrigiano G (2011) Too many cooks? Intrinsic and synaptic homeostatic mechanisms in cortical circuit refinement. Annu Rev Neurosci 34:89-103. CrossRef

Tyagarajan SK, Ghosh H, Yevenes GE, Nikonenko I, Ebeling C, Schwerdel C, Sidler C, Zeilhofer HU, Gerrits B, Muller D, Fritschy JM (2011) Regulation of GABAergic synapse formation and plasticity by GSK3beta-dependent phosphorylation of gephyrin. Proc Natl Acad Sci U S A 108:379-384. CrossRef

Ulanovsky N, LAS L, Nelken I (2003) Processing of low-probability sounds by cortical neurons. Nat Neurosci 6:391-398. CrossRef Medline

Voss P, Thomas M, Chou YC, Cisneros-Franco JM, Ouellet L, DE Villers-Sidani E (2016) Pairing cholinergic enhancement with perceptual training promotes recovery of age-related changes in rat primary auditory cortex. Neural Plast 2016:1801979. CrossRef

Voss P, Thomas ME, Cisneros-Franco JM, DE Villers-Sidani E (2017) Dynamic brains and the changing rules of neuroplasticity: implications for learning and recovery. Front Psychol 8:1657. CrossRef

Wang D, Fawcett J (2012) The perineuronal net and the control of CNS plasticity. Cell Tissue Res 349:147-160. CrossRef Medline

Yamada J, Ohgomori T, Jinno S (2015) Perineuronal nets affect parvalbumin expression in GABAergic neurons of the mouse hippocampus. Eur J Neurosci 41:368-378. CrossRef

Zhou X, DE Villers-Sidani E, Panizzutti R, Merzenich MM (2010) Successive-signal biasing for a learned sound sequence. Proc Natl Acad Sci U S A 107:14839-14844. CrossRef Medline

Zhou X, Panizzutti R, DE Villers-Sidani E, Madeira C, Merzenich MM (2011) Natural restoration of critical period plasticity in the juvenile and adult primary auditory cortex. J Neurosci 31:5625-5634. CrossRef

Zhou X, Merzenich MM (2012) Environmental noise exposure degrades normal listening processes. Nat Commun 3:843. CrossRef 